\title{
Self-Condensation of Pyrene-4,5-dione: An Approach to Generate Functional Organic Fluorophores
}

\author{
Farshid Shahrokhi and Yuming Zhao* \\ Department of Chemistry, Memorial University of Newfoundland \\ St. John's, NL, Canada A1B 3X7 \\ Email: yuming@mun.ca
}

\section{Table of Content}

1. Experimental $\quad$ S-2

2. NMR Spectra for New Compounds S-6

3. UV-Vis Spectroscopic Data S-14

4. Photographic Images $\quad$ S-18

5. Cyclic Voltammetric Data of 2b S-19

6. Detailed Results of DFT Calculations S-20

7. X-ray Structure of $\left[\mathbf{2 b} \cdot(\mathrm{TFA})_{2}\right] \quad$ S-32

\section{Experimental}




\subsection{General}

Chemicals were purchased from commercial suppliers and used directly without purification. All reactions were conducted in standard glassware. Evaporation and concentration were carried out with a rotary evaporator. Flash column chromatography was performed with 240-400 mesh silica gel, and thin-layer chromatography (TLC) was carried out with silica gel F254 covered on plastic sheets and visualized by UV light. Melting points (m.p.) were measured using an SRS OptiMelt melting point apparatus and are uncorrected. ${ }^{1} \mathrm{H}$ and ${ }^{13} \mathrm{C}$ NMR spectra were measured on a Bruker Avance III $300 \mathrm{MHz}$ and Bruker Avance $500 \mathrm{MHz}$ multinuclear spectrometer. Chemical shifts $(\delta)$ are reported in ppm downfield relative to the signals of the internal reference $\mathrm{SiMe}_{4}$ or residual solvents $\left(\mathrm{CHCl}_{3}: \delta_{\mathrm{H}}=7.24 \mathrm{ppm}, \delta_{\mathrm{C}}=77.2 \mathrm{ppm} ; \mathrm{CH}_{2} \mathrm{Cl}_{2}: \delta_{\mathrm{H}}=5.32 \mathrm{ppm}, \delta_{\mathrm{C}}=54.0 \mathrm{ppm}\right)$. Coupling constants $(J)$ are given in Hz. Infrared spectra (IR) were recorded on a Bruker Alfa spectrometer. MALDI-TOF MS analysis was performed on Bruker 9.4T Apex-Qe FTICR system. Highresolution APPI-TOF MS analysis was done on a GCT premier Micromass Technologies instrument. UV-Vis absorption spectra were measured on a Cary 6000i spectrophotometer. Fluorescence spectra were measured on a Photon Technology International (PTI) QuantaMaster spectrofluorometer. Relative fluorescence quantum yields $\left(\phi_{\mathrm{F}}\right)$ in solution were determined following reported procedures ${ }^{1}$ using quninine sulfate $\left(\phi_{\mathrm{F}}=0.546\right)$ as the standard.

Single crystal X-ray diffraction analysis was conducted on a Bruker PLATFORM/APEX II CCD diffractometer equipped with a $\mathrm{Cu}-K \alpha(1.54178 \AA)$ source. The crystal structures were solved and refined with the SHELXT software package.

Density functional theory (DFT) calculations were performed using the Gaussian 16 software package. Geometry optimization was done at the M06-2X/6-31G(d) level and the optimized geometries were further confirmed by frequency calculations at the same level of theory as energy minima (zero imaginary frequency) or transition state (one imaginary frequency). NMR calculations were carried out using the Gauge-Independent Atomic Orbital (GIAO) method at the B3LYP/6-31+G(2d,p) level of theory with the solvent effect (methylene chloride) taken into account by the polarizable continuum model (PCM).

\subsection{Synthesis}

\section{$6 H$-phenanthro[4,5-cde]pyreno $\left[4^{\prime}, 5^{\prime}: 4,5\right]$ imidazo[1,2-a] azepin-6-one (2a)}
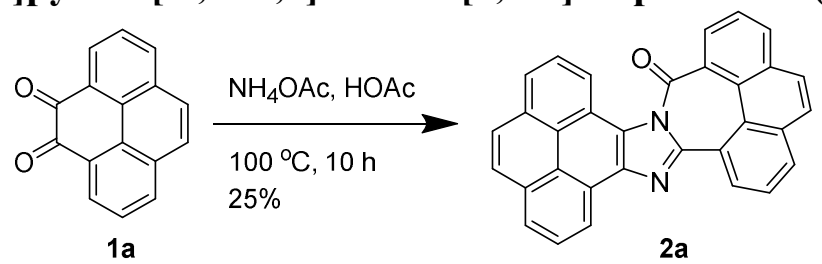

Pyrene-4,5-dione (1a) (0.090 g, $0.387 \mathrm{mmol})$, ammonium acetate $(0.239 \mathrm{~g}, 3.103 \mathrm{mmol})$, and glacial acetic acid $(99.7 \%, 5 \mathrm{~mL})$ were added to a $50 \mathrm{~mL}$ round-bottomed flask. The reaction

\footnotetext{
${ }^{1}$ Williams, A. T. R.; Winfield, S. A.; Miller, J. N. Analyst, 1983, 108, 1067-1071; Miller, J. N. in Standards in Flourescence Spectrometry. Techniques in Visible and Ultraviolet Spectometry, Vol 2., Miller, J. N., Ed.; Springer, Dordrecht, 1981, pp 68-78.
} 
mixture was stirred and heated at $100{ }^{\circ} \mathrm{C}$ for $10 \mathrm{~h}$. Next, the reaction mixture was cooled down to room temperature. The resulting yellow solid was collected by suction filtration and washed sequentially with a saturated aqueous $\mathrm{NaHCO}_{3}$ solution, water, and acetone. The resulting residue was washed with $\mathrm{CH}_{2} \mathrm{Cl}_{2}(2 \mathrm{~mL})$ to give compound $\mathbf{2 a}(0.043 \mathrm{~g}, 0.097 \mathrm{mmol}, 25 \%)$ as a yellow solid. m.p. $>386.1{ }^{\circ} \mathrm{C}$ (dec); IR (neat): 2958, 2921, 2851, 1692, 1622, 1586, 1464, 1337, 1320, 1255, 1151, 823, $717 \mathrm{~cm}^{-1} ;{ }^{1} \mathrm{H}$ NMR $\left(300 \mathrm{MHz} \mathrm{CDCl}_{3}\right): \delta 9.52(\mathrm{dd}, J=7.7,1.5 \mathrm{~Hz}, 1 \mathrm{H}), 9.16$ $(\mathrm{dd}, J=7.5,1.2 \mathrm{~Hz}, 1 \mathrm{H}), 8.74(\mathrm{dd}, J=7.5,1.5 \mathrm{~Hz}, 1 \mathrm{H}), 8.61-8.56(\mathrm{~m}, 1 \mathrm{H}), 8.34$ (dd, $J=7.9,1.6$ $\mathrm{Hz}, 1 \mathrm{H}), 8.30-7.95(\mathrm{~m}, 11 \mathrm{H})$; Meaningful ${ }^{13} \mathrm{C}$ NMR spectrum could not be obtained due to limited solubility; HRMS (APPI-TOF, positive mode) $m / z$ calcd for $\mathrm{C}_{32} \mathrm{H}_{16} \mathrm{~N}_{2} \mathrm{O} 444.1263$, found 444.1273 $[\mathrm{M}+\mathrm{H}]^{+}$.

\section{4,9,14,18-Tetra-tert-butyl-6H-phenanthro[4,5-cde]pyreno $\left[4^{\prime}, 5^{\prime}: 4,5\right]$ imidazo $[1,2-a]$ azepin-6- one (2b)}

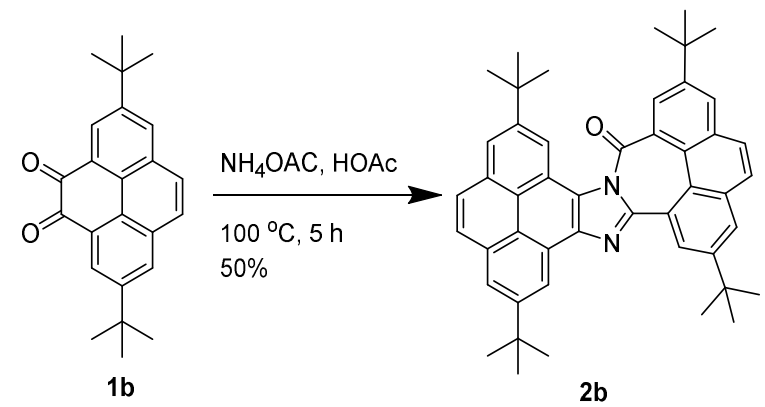

2,7-Di-tert-butylpyrene-4,5-dione (1b) (0.344 g, $1.00 \mathrm{mmol})$, ammonium acetate (0.616 g, 8.00 $\mathrm{mmol})$, and glacial acetic acid $(99.7 \%, 5 \mathrm{~mL})$ were added to a $50 \mathrm{~mL}$ round-bottomed flask. The reaction mixture was stirred and heated at $100{ }^{\circ} \mathrm{C}$ for $5 \mathrm{~h}$. Next, the reaction mixture was cooled down to room temperature and then poured into a separatory funnel including water. The mixture was extracted twice with $\mathrm{CH}_{2} \mathrm{Cl}_{2}$. The combined organic layer was washed twice with a saturated aqueous $\mathrm{NaHCO}_{3}$ solution. The organic layer was dried over $\mathrm{MgSO}_{4}$, filtered, and concentrated in vacuo. The resulting residue was subjected to silica gel column chromatography (hexanes/ $\left.\mathrm{CH}_{2} \mathrm{Cl}_{2}, 80: 20\right)$ to give pure compound $\mathbf{2 b}(0.219 \mathrm{~g}, 0.327 \mathrm{mmol}, 50 \%)$ as a yellow crystalline solid. m.p. $>254.6{ }^{\circ} \mathrm{C}$ (dec.); IR (neat): 3051, 2950, 2901, 2863, 1696, 1605, 1472, 1433, 1393, 1262, 1204, 1073, $891 \mathrm{~cm}^{-1} ;{ }^{1} \mathrm{H}$ NMR $\left(300 \mathrm{MHz}, \mathrm{CD}_{2} \mathrm{Cl}_{2}\right): \delta 9.63(\mathrm{~d}, J=2.3 \mathrm{~Hz}, 1 \mathrm{H}), 9.18(\mathrm{~d}, J=$ $1.9 \mathrm{~Hz}, 1 \mathrm{H}), 8.76(\mathrm{~d}, J=2.3 \mathrm{~Hz}, 1 \mathrm{H}), 8.64(\mathrm{~d}, J=1.8 \mathrm{~Hz}, 1 \mathrm{H}), 8.34(\mathrm{~d}, J=2.3 \mathrm{~Hz}, 1 \mathrm{H}), 8.31(\mathrm{~d}, J$ $=2.0 \mathrm{~Hz}, 1 \mathrm{H}), 8.26(\mathrm{~d}, J=1.8 \mathrm{~Hz}, 1 \mathrm{H}), 8.21(\mathrm{~d}, J=2.3 \mathrm{~Hz}, 1 \mathrm{H}), 8.11(\mathrm{~s}, 2 \mathrm{H}), 7.97(\mathrm{~d}, J=1.5 \mathrm{~Hz}$, $2 \mathrm{H}), 1.73(\mathrm{~s}, 9 \mathrm{H}), 1.69(\mathrm{~s}, 9 \mathrm{H}) 1.67(\mathrm{~s}, 9 \mathrm{H}) 1.63(\mathrm{~s}, 9 \mathrm{H}) ;{ }^{13} \mathrm{C} \mathrm{NMR}\left(75 \mathrm{MHz}, \mathrm{CD}_{2} \mathrm{Cl}_{2}\right): \delta 171.76$, $150.24,150.08,149.86,149.43,148.22$, 140.93, 133.74, 133.47, 132.77, 131.83, 131.42, 130.18, $129.44,128.99$, 128.56, 128.12, 127.93, 127.67, 127.60, 127.08, 125.54, 125.14, 124.37, 123.71, 122.66, 122.02, 121.90, 121.87, 121.41, 118.41, 117.79, 35.53, 35.39, 35.04, 34.97, 31.88, 31.74, 31.17, 31.08; HRMS (APPI-TOF, positive mode) $\mathrm{m} / z$ calcd for $\mathrm{C}_{48} \mathrm{H}_{48} \mathrm{~N}_{2} \mathrm{O} 668.3767$, found $668.3783[\mathrm{M}+\mathrm{H}]^{+}$. X-ray. 


\section{4,18-Di-tert-butyl-6H-phenanthro[4,5-cde]pyreno[4',5':4,5]imidazo[1,2-a]azepin-6-one (2c)}
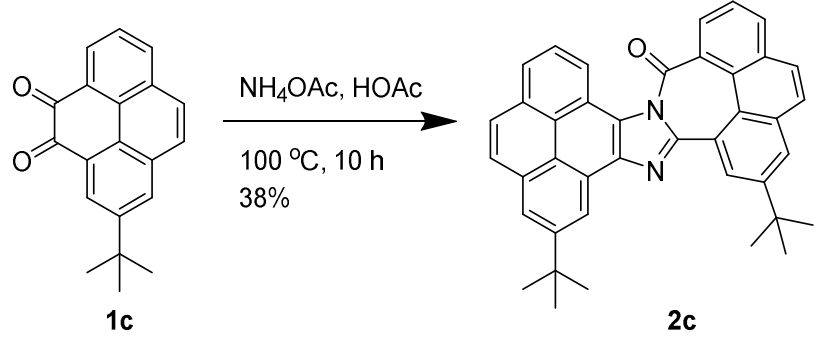

2-(tert-Butyl)pyrene-4,5-dione (1c) $(0.060 \mathrm{~g}, 0.208 \mathrm{mmol})$, ammonium acetate $(0.128 \mathrm{~g}, 1.664$ $\mathrm{mmol})$, and glacial acetic acid $(99.7 \%, 5 \mathrm{~mL})$ were added to a $50 \mathrm{~mL}$ round-bottomed flask. The reaction mixture was stirred and heated at $100^{\circ} \mathrm{C}$ for $10 \mathrm{~h}$. Next, the reaction mixture was cooled down to room temperature and then poured into a separatory funnel including water. The mixture was extracted twice with $\mathrm{CH}_{2} \mathrm{Cl}_{2}$. The combined organic layer was washed twice with a saturated aqueous $\mathrm{NaHCO}_{3}$ solution. The organic layer was dried over $\mathrm{MgSO}_{4}$, filtered, and concentration in vacuo. The resulting residue was subjected to silica gel column chromatography (hexanes $\left./ \mathrm{CH}_{2} \mathrm{Cl}_{2} / \mathrm{EtOAC}, 80: 20: 3\right)$ to give pure compound $\mathbf{2 c}(0.044 \mathrm{~g}, 0.076 \mathrm{mmol}, 38 \%$ ) as a yellow crystalline solid: m.p. $246.3-247.9^{\circ} \mathrm{C}$. IR (neat): 3051, 2950, 2902, 2863, 1687, 1602, $1567,1475,1316,1261,1202,1055,891 \mathrm{~cm}^{-1} ;{ }^{1} \mathrm{H}$ NMR $\left(300 \mathrm{MHz}, \mathrm{CD}_{2} \mathrm{Cl}_{2}\right): \delta 9.62(\mathrm{~d}, J=2.3$ $\mathrm{Hz}, 1 \mathrm{H}), 9.23(\mathrm{~d}, J=2.0 \mathrm{~Hz}, 1 \mathrm{H}), 8.70(\mathrm{dd}, J=7.5,1.5 \mathrm{~Hz}, 1 \mathrm{H}), 8.53(\mathrm{dd}, J=7.9,1.1 \mathrm{~Hz}, 1 \mathrm{H})$, 8.36-8.32 (m, 2H), 8.24-8.20 (m, 2H), 8.14-8.08 (m, 3H), 8.02-7.93 (m, 3H), $1.69(\mathrm{~s}, 9 \mathrm{H}), 1.67$ (s, 9H); ${ }^{13} \mathrm{C}$ NMR $\left(75 \mathrm{MHz}, \mathrm{CD}_{2} \mathrm{Cl}_{2}\right): \delta 171.89,151.21,150.71,150.40,141.52,134.45,134.32$, $133.77,133.45,132.59,132.17,131.30,130.35,129.86,128.77,128.34,128.28,127.95,127.37$, $127.22,126.31,125.96,125.87,125.50,125.02,124.30,123.45,122.48,122.32,121.03,118.52$, 35.97, 35.63, 32.26, 31.70; HRMS (APPI-TOF, positive mode) $m / z$ calcd for $\mathrm{C}_{40} \mathrm{H}_{32} \mathrm{~N}_{2} \mathrm{O} 556.2514$, found $556.2512[\mathrm{M}+\mathrm{H}]^{+}$. X-ray.

\section{4,18-Di-tert-butyl-6H-phenanthro[4,5-cde]phenanthro $\left[9^{\prime}, 10^{\prime}: 4,5\right]$ imidazo[1,2-a] azepin-6- one (5)}

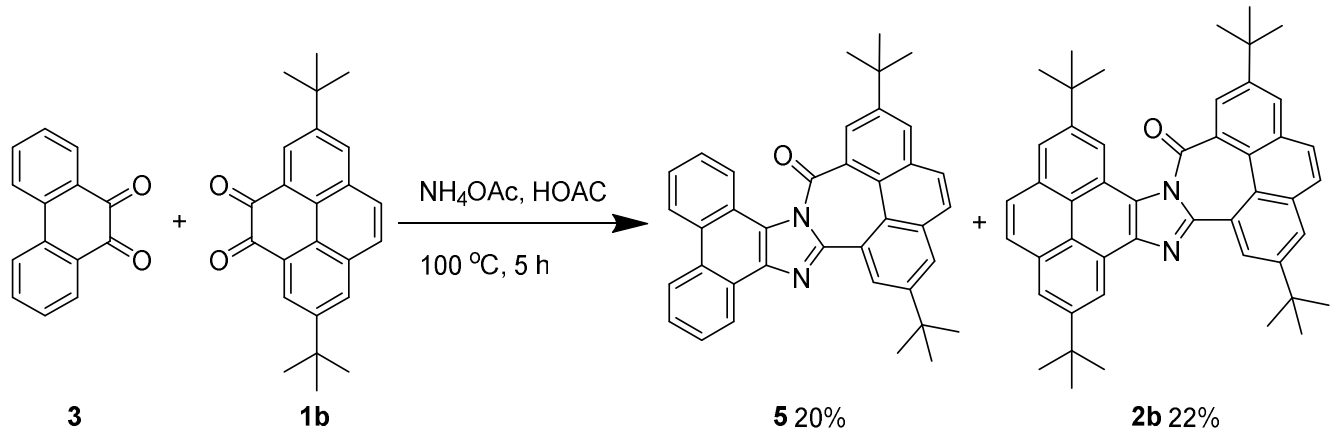

2,7-Di-tert-butylpyrene-4,5-dione (1b) (0.050 g, $0.17 \mathrm{mmol})$, phenanthrene-9,10-dione (3) (0.050 $\mathrm{g}, 0.24 \mathrm{mmol})$, ammonium acetate $(0.107 \mathrm{~g}, 1.392 \mathrm{mmol})$, and glacial acetic acid $(99.7 \%, 5 \mathrm{~mL})$ were added to a $50 \mathrm{~mL}$ round-bottomed flask. The reaction mixture was stirred and heated at 100 ${ }^{\circ} \mathrm{C}$ for $5 \mathrm{~h}$. Next, the mixture was cooled down to room temperature and then poured into a separatory funnel including water. The mixture was extracted twice with $\mathrm{CH}_{2} \mathrm{Cl}_{2}$. The combined organic layer washed twice with a saturated aqueous $\mathrm{NaHCO}_{3}$ solution. The organic layer was dried over $\mathrm{MgSO}_{4}$, filtered, and concentrated in vacuo. The resulting residue was subjected to 
silica gel column chromatography (hexanes $\left./ \mathrm{CH}_{2} \mathrm{Cl}_{2}, 80: 20\right)$ to give pure compound $\mathbf{5}(0.018 \mathrm{~g}$, $0.035 \mathrm{mmol}, 20 \%)$ and compound $2 \mathrm{~b}(0.022 \mathrm{~g}, 0.032 \mathrm{mmol}, 22 \%)$. Both products appeared as yellow crystalline solids. Compound 5: m.p. $>251.2{ }^{\circ} \mathrm{C}$ (dec.); IR (neat): 3058, 2952, 2924, 2855 , 1687, 1608, 1567, 1478, 1362, 1261, 1224, 1076, $888 \mathrm{~cm}^{-1}$; ${ }^{1} \mathrm{H}$ NMR $\left(300 \mathrm{MHz}, \mathrm{CD}_{2} \mathrm{Cl}_{2}\right): \delta 9.56$ $(\mathrm{d}, J=2.3 \mathrm{~Hz}, 1 \mathrm{H}), 8.92(\mathrm{~m}, 1 \mathrm{H}), 8.86(\mathrm{~m}, 1 \mathrm{H}), 8.78(\mathrm{~m}, 1 \mathrm{H}), 8.67(\mathrm{~d}, J=2.3 \mathrm{~Hz}, 1 \mathrm{H}), 8.30(\mathrm{~d}, J$ $=2.3 \mathrm{~Hz}, 1 \mathrm{H}), 8.27(\mathrm{~m}, 1 \mathrm{H}), 8.18(\mathrm{~d}, J=2.3 \mathrm{~Hz}, 1 \mathrm{H}), 7.94(\mathrm{~d}, J=1.1 \mathrm{~Hz}, 2 \mathrm{H}), 7.82-7.67(\mathrm{~m}, 4 \mathrm{H})$, $1.63(\mathrm{~s}, 9 \mathrm{H}), 1.61(\mathrm{~s}, 9 \mathrm{H}) ;{ }^{13} \mathrm{C}$ NMR $\left(75 \mathrm{MHz}, \mathrm{CD}_{2} \mathrm{Cl}_{2}\right): \delta 172.11,150.76,150.72,150.45,140.86$, $134.31,134.00,133.05,130.77,130.72,130.39,130.07,129.52,129.39,128.72,128.27,127.88$, $127.32,127.06,126.91,126.17,125.99,124.92,124.70,124.24,123.94,123.91,123.81,123.52$, $35.55,31.68,31.61,30.28$; HRMS (APPI-TOF, positive mode) $m / z$ calcd for $\mathrm{C}_{38} \mathrm{H}_{32} \mathrm{~N}_{2} \mathrm{O} 532.2515$, found $532.2526[\mathrm{M}+\mathrm{H}]^{+}$. X-ray.

\section{2'-(1H-phenanthro[9,10-d]imidazol-2-yl)-[1,1'-biphenyl]-2-carboxylic acid (4)}

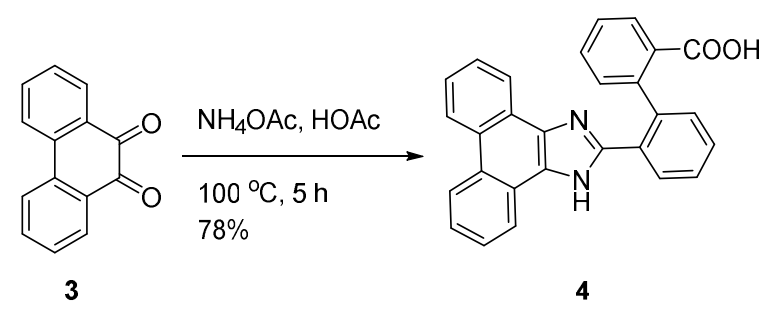

Phenanthrene-9,10-dione (3) (0.200 g, $0.960 \mathrm{mmol})$, ammonium acetate $(0.592 \mathrm{~g}, 7.684 \mathrm{mmol})$, and glacial acetic acid $(99.7 \%, 10 \mathrm{~mL})$ were added to a $50 \mathrm{~mL}$ round-bottomed flask. The reaction mixture was stirred and heated at $100{ }^{\circ} \mathrm{C}$ for $5 \mathrm{~h}$. Next, the mixture was cooled down to room temperature and kept at this temperature for 2 days. Compound $4(0.310 \mathrm{~g}, 0.749 \mathrm{mmol}, 78 \%)$ was collected as a pale yellow solid after vacuum filtration and solvent rinsing. The structure of 4 was confirmed by ${ }^{1} \mathrm{H}$ NMR, which is consistent with the data reported by Lantos [see, J. Org. Chem. 1975, 40, 1641-1642]. 


\section{NMR Spectra for New Compounds}

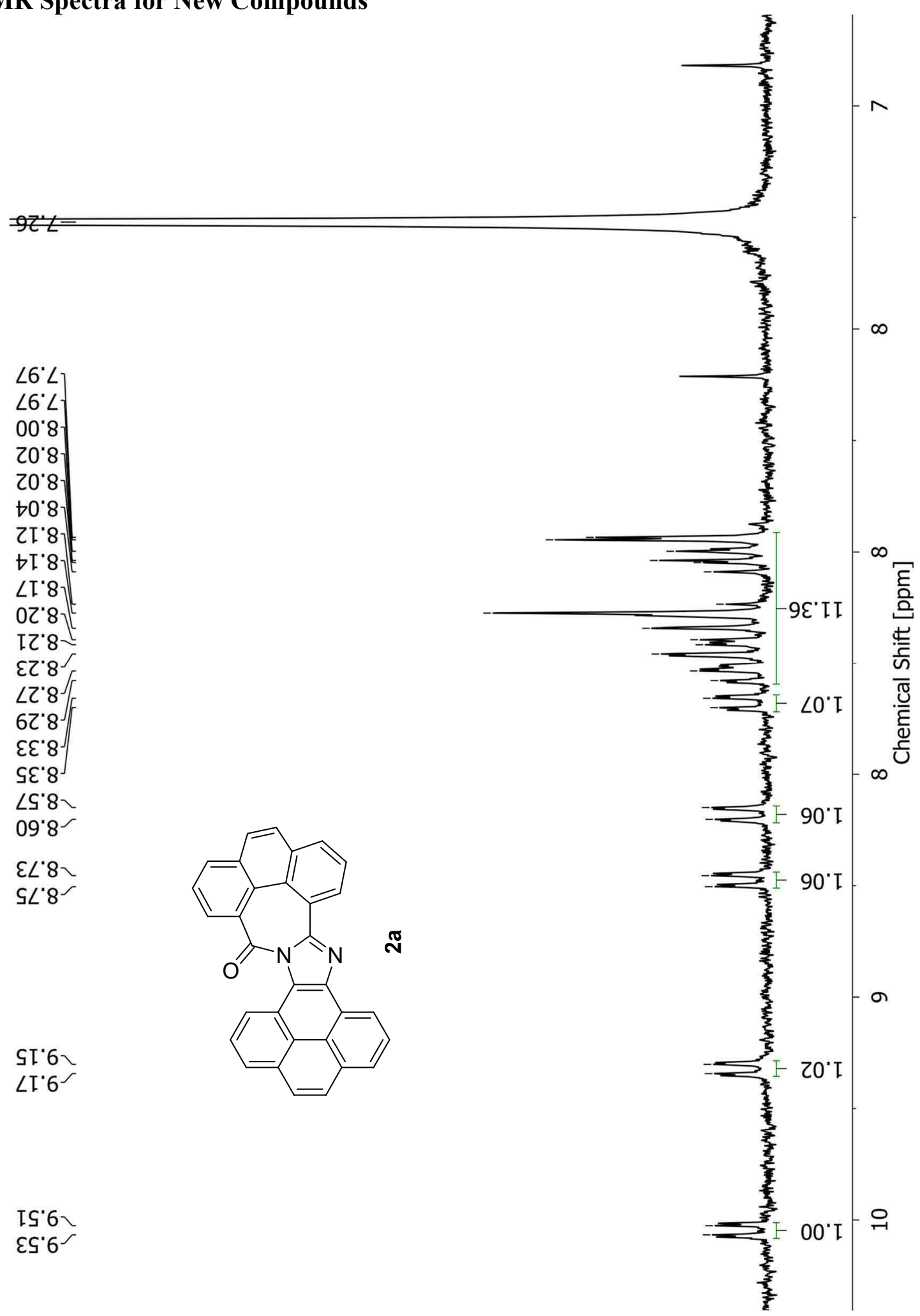

Fig. S-1 ${ }^{1} \mathrm{H}$ NMR (300 MHz, $\left.\mathrm{CD}_{2} \mathrm{Cl}_{2}\right)$ of compound $2 \mathbf{a}$. 


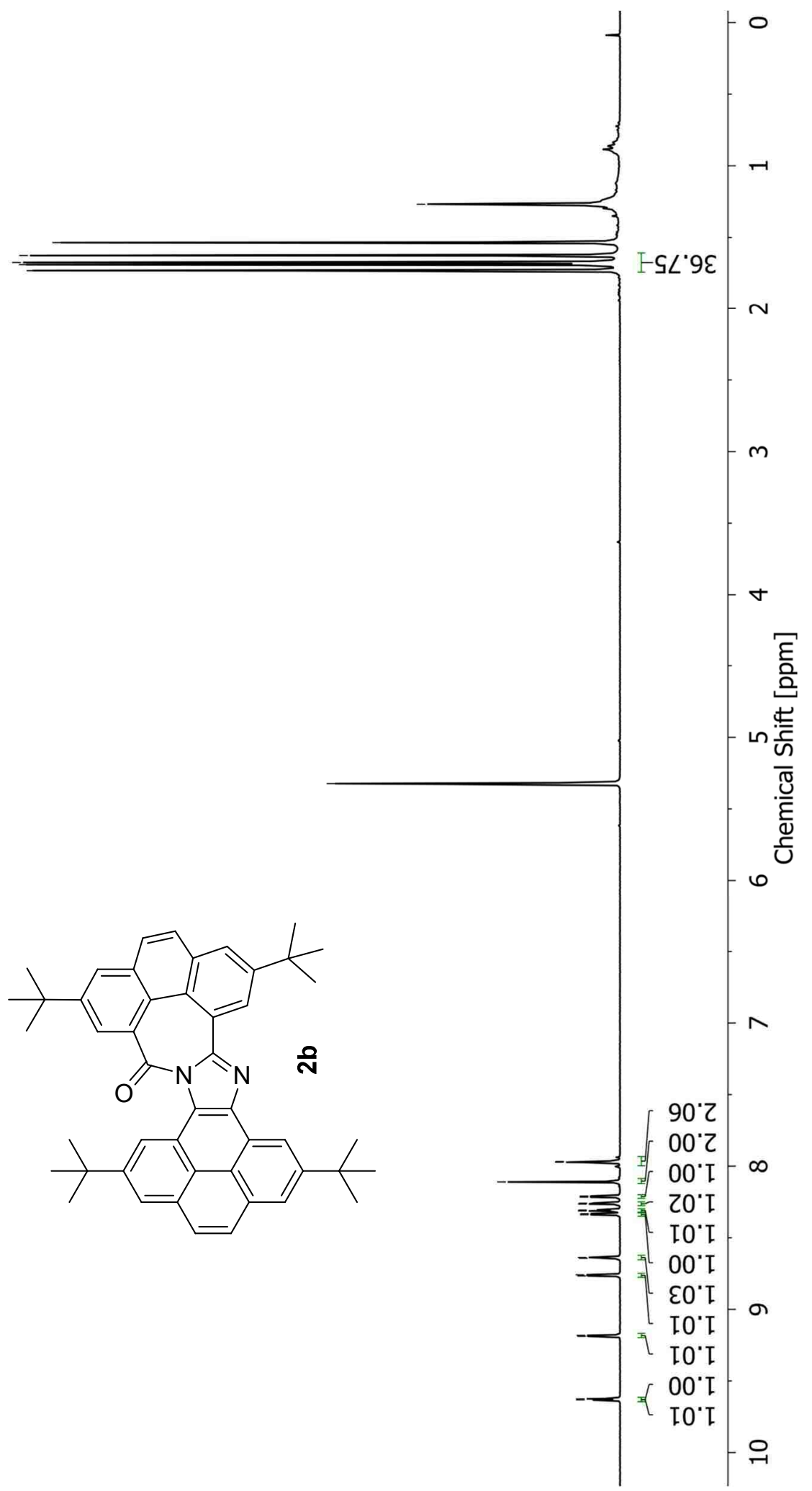

Fig. S-2 ${ }^{1} \mathrm{H}$ NMR (300 MHz, $\left.\mathrm{CD}_{2} \mathrm{Cl}_{2}\right)$ of compound $\mathbf{2 b}$. 


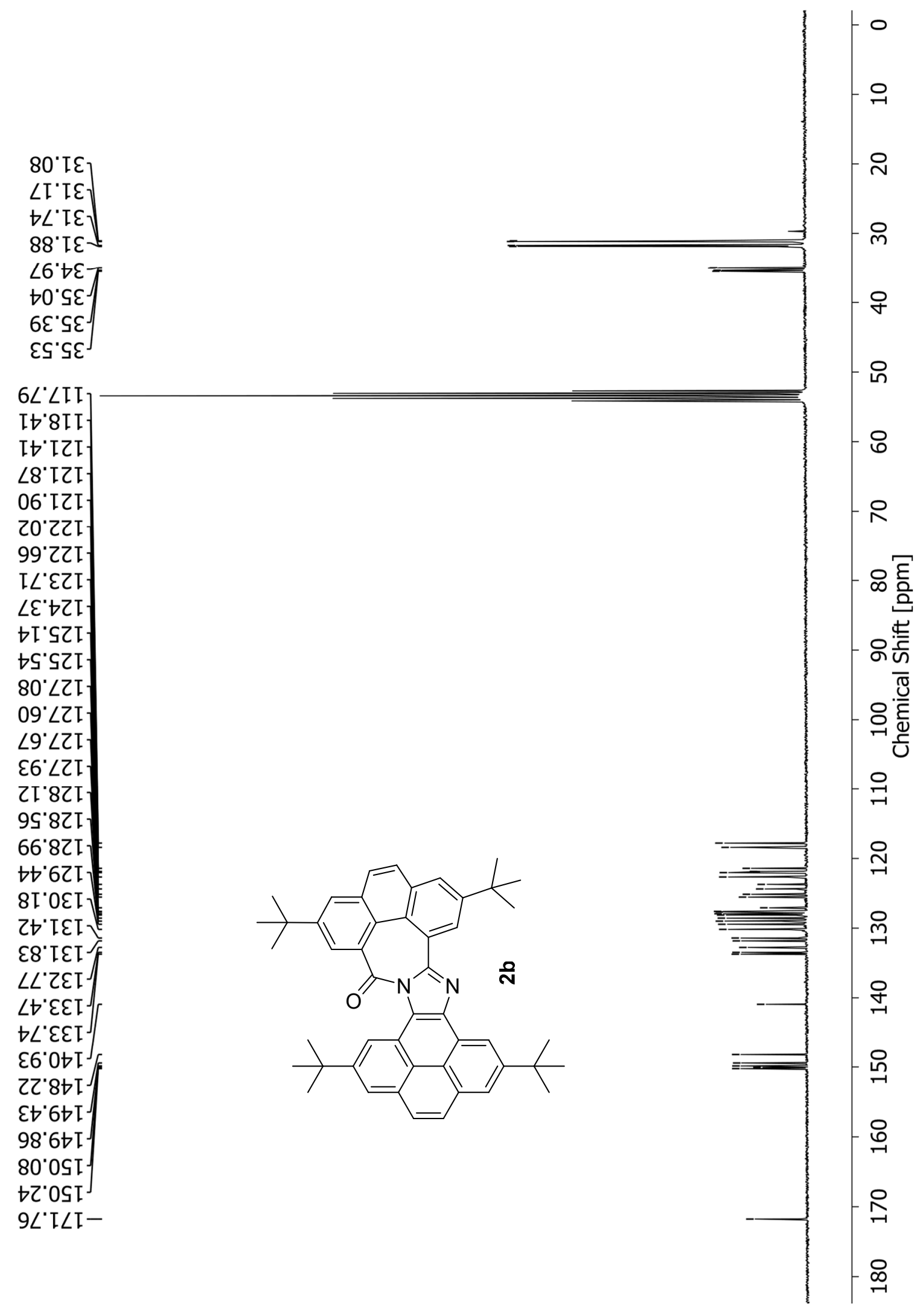

Fig. S-3 ${ }^{13} \mathrm{C}$ NMR (75 MHz, $\left.\mathrm{CD}_{2} \mathrm{Cl}_{2}\right)$ of compound $\mathbf{2 b}$. 


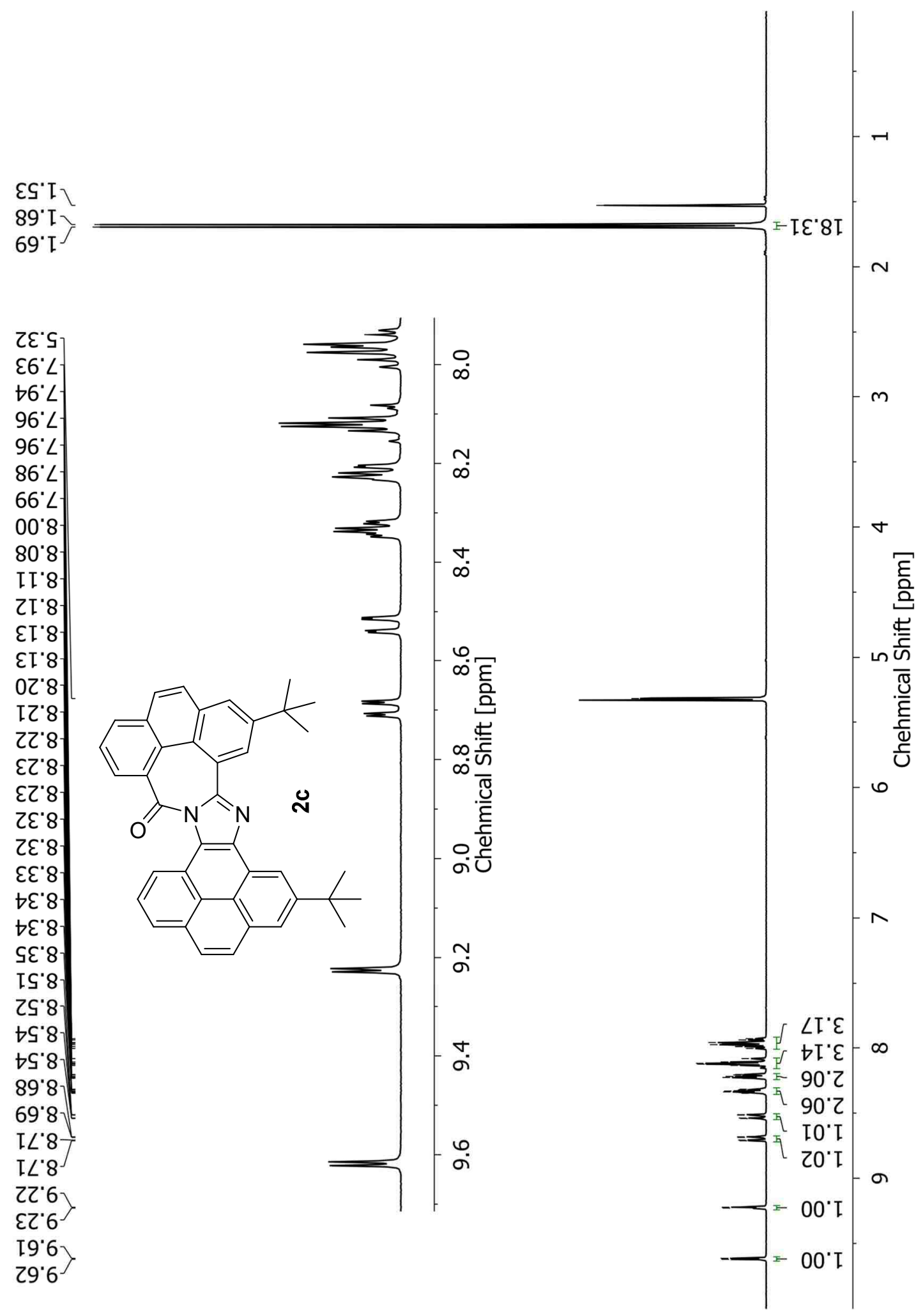

Fig. S-4 ${ }^{1} \mathrm{H}$ NMR (300 MHz, $\left.\mathrm{CD}_{2} \mathrm{Cl}_{2}\right)$ of compound 2c. 


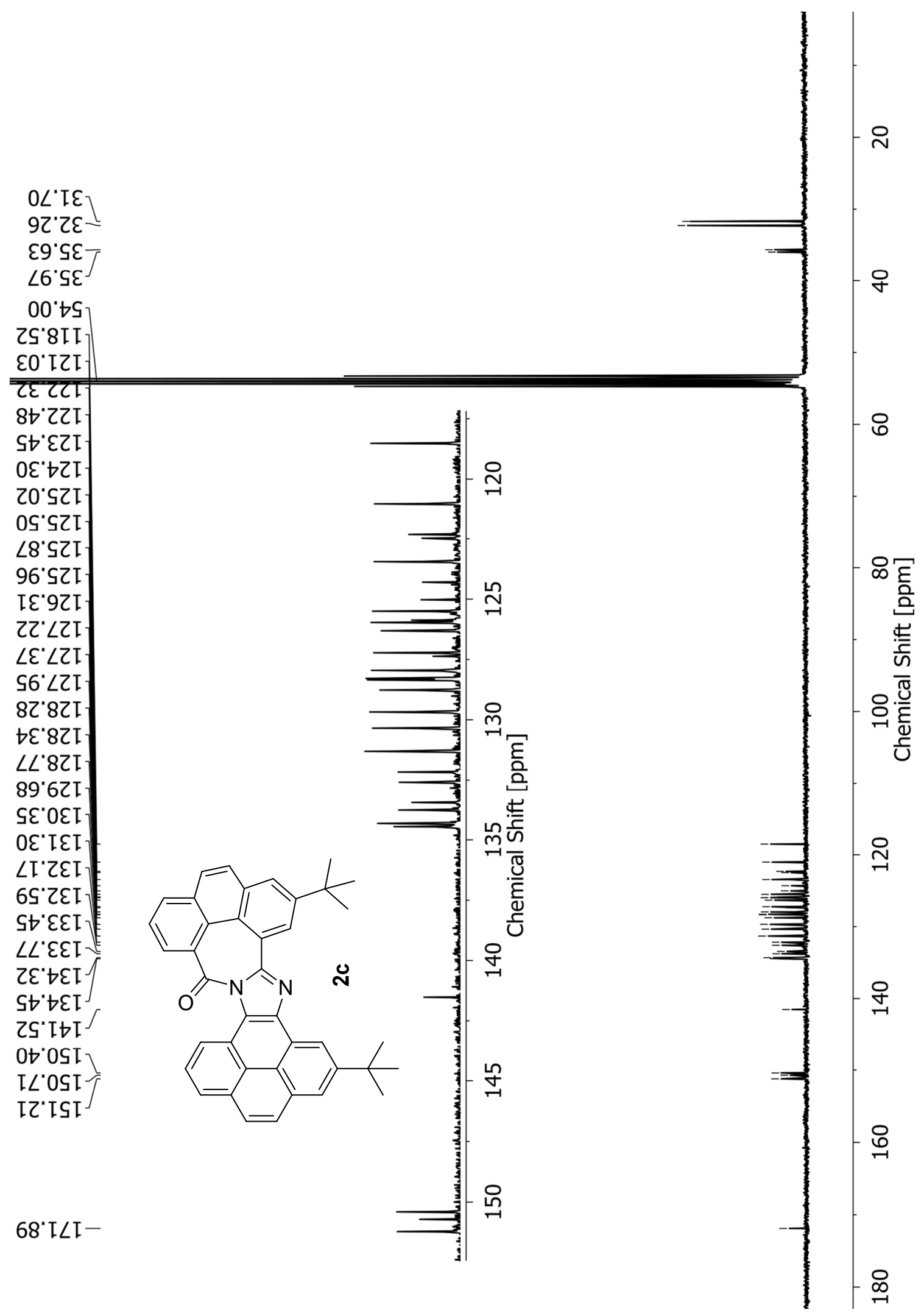

Fig. S-5 ${ }^{13} \mathrm{C}$ NMR (75 MHz, $\left.\mathrm{CD}_{2} \mathrm{Cl}_{2}\right)$ of compound 2c. 


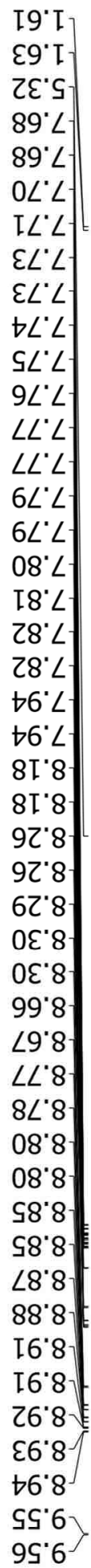

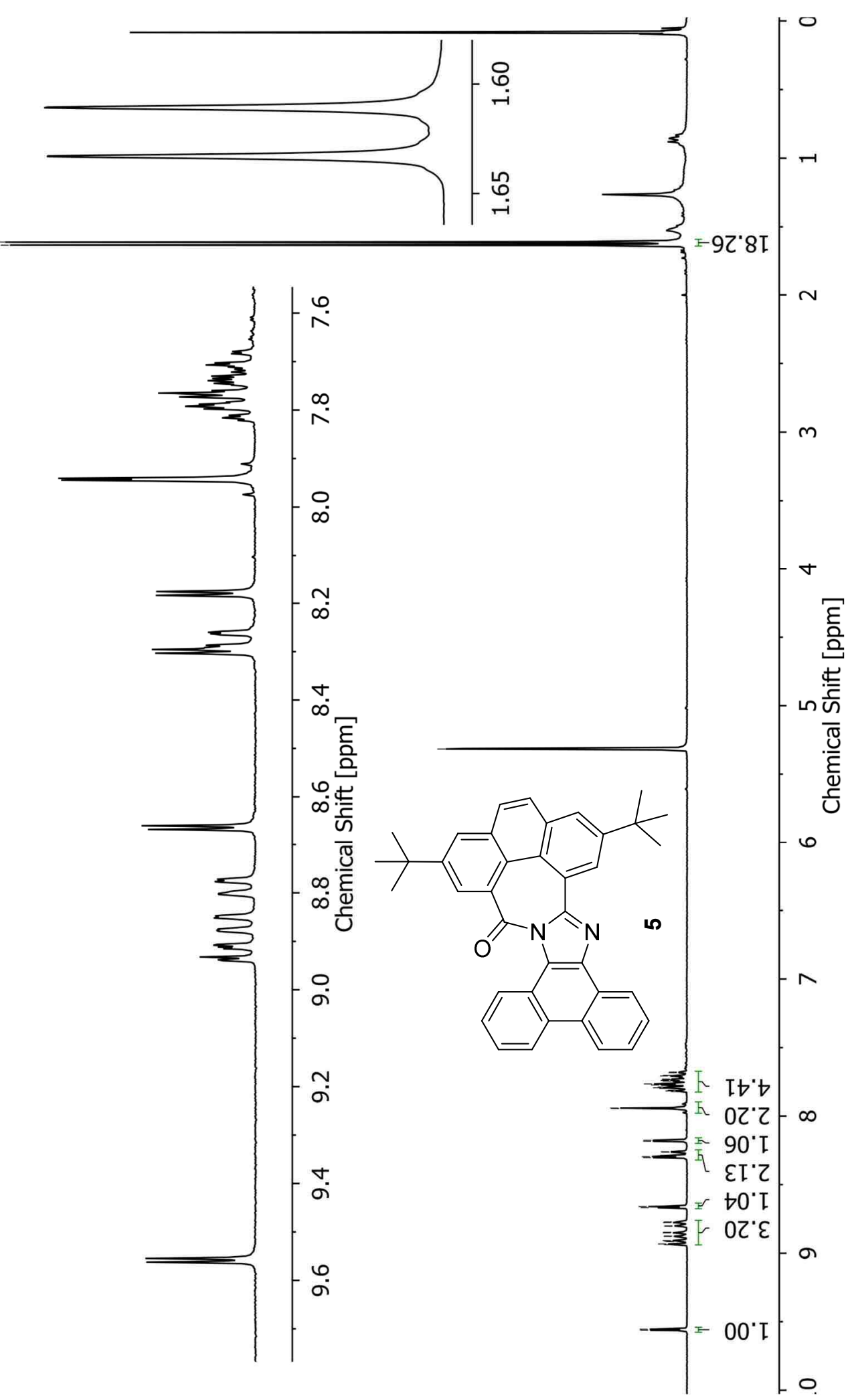

Fig. S-6 ${ }^{1} \mathrm{H}$ NMR $\left(300 \mathrm{MHz}, \mathrm{CD}_{2} \mathrm{Cl}_{2}\right)$ of compound 5. 


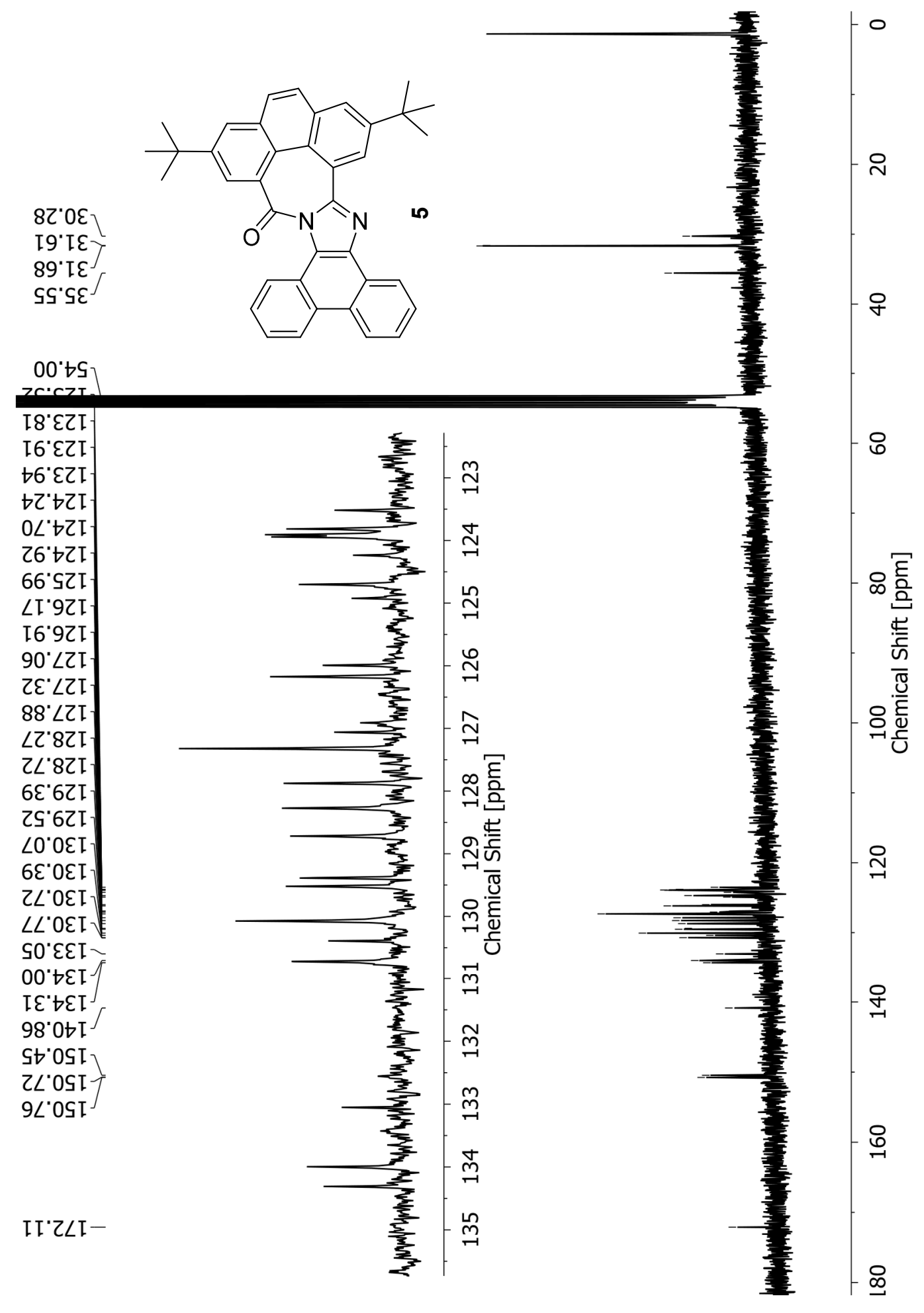

Fig. S-7 ${ }^{13} \mathrm{C}$ NMR (75 MHz, $\left.\mathrm{CD}_{2} \mathrm{Cl}_{2}\right)$ of compound 5. 


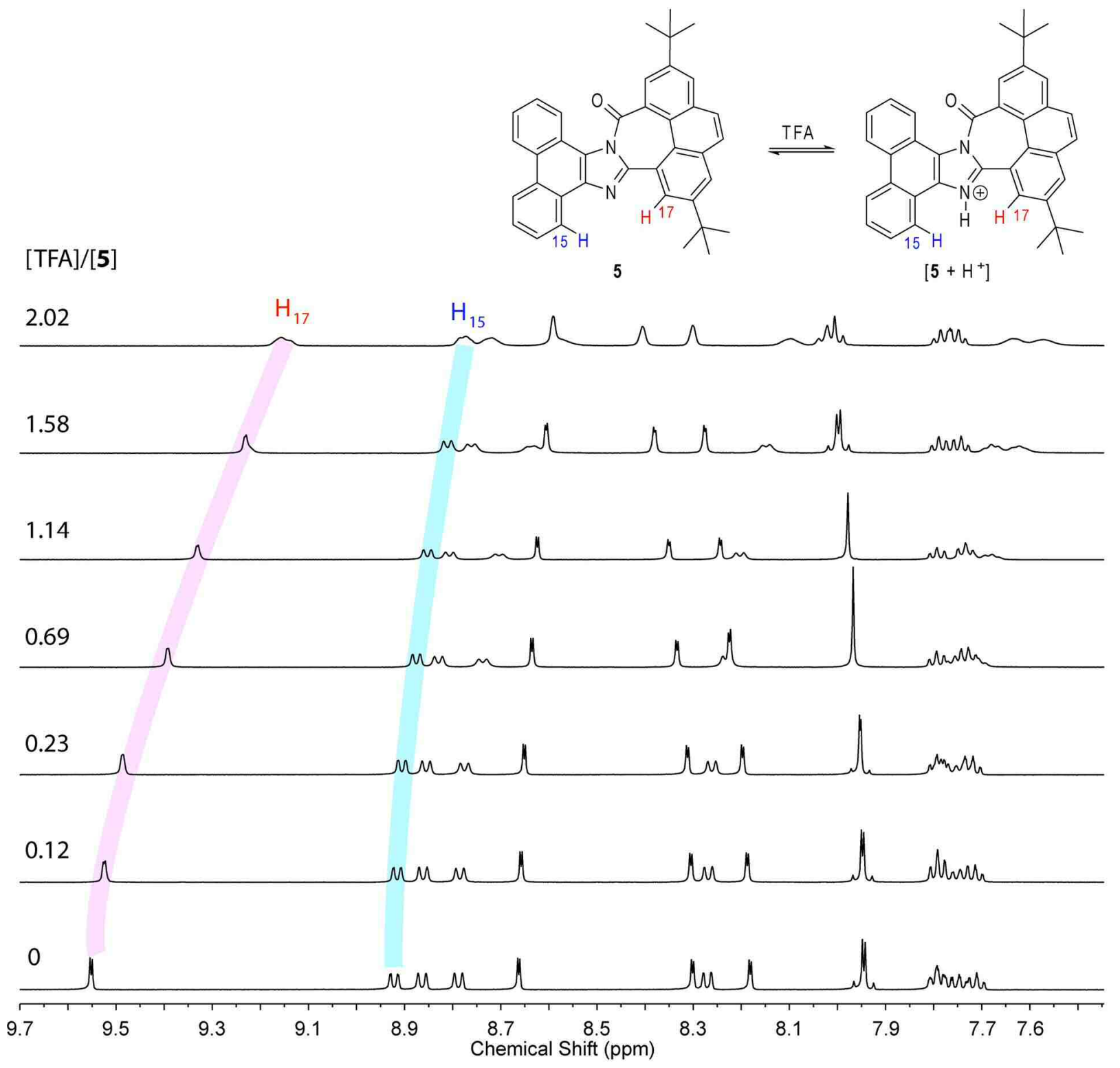

Fig. S-8 ${ }^{1} \mathrm{H}$ NMR spectra of $5\left(8.04 \times 10^{-3} \mathrm{M}\right.$ in $\left.\mathrm{CD}_{2} \mathrm{Cl}_{2}\right)$ in response to the addition of TFA from 0 to 2.02 molar equivalents. 


\section{UV-Vis Spectroscopic Data}

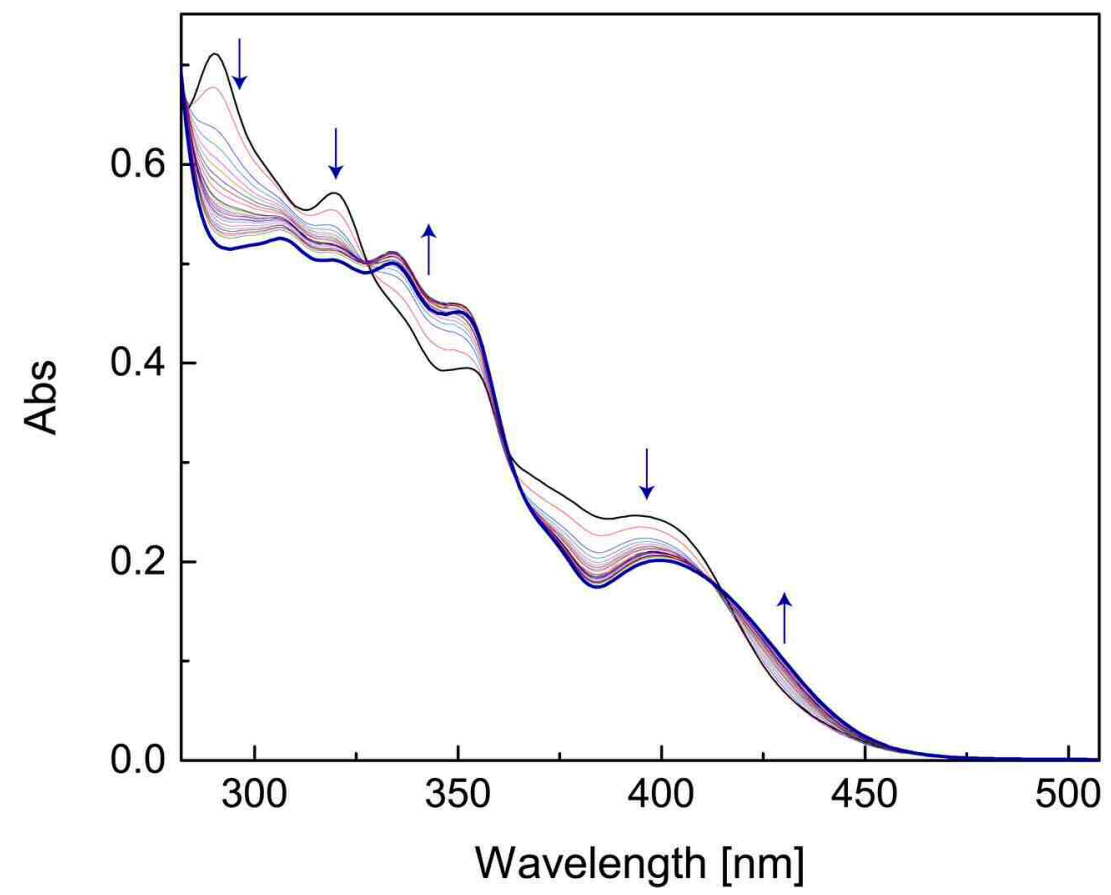

Fig. S-9. UV-Vis absorption spectra of $\mathbf{2 b}\left(1.49 \times 10^{-5} \mathrm{M}\right.$ in carbon tetrachloride $)$ in response to the addition of TFA from 0 to $3.10 \times 10^{3}$ molar equivalents. Arrows indicate the trend of change.

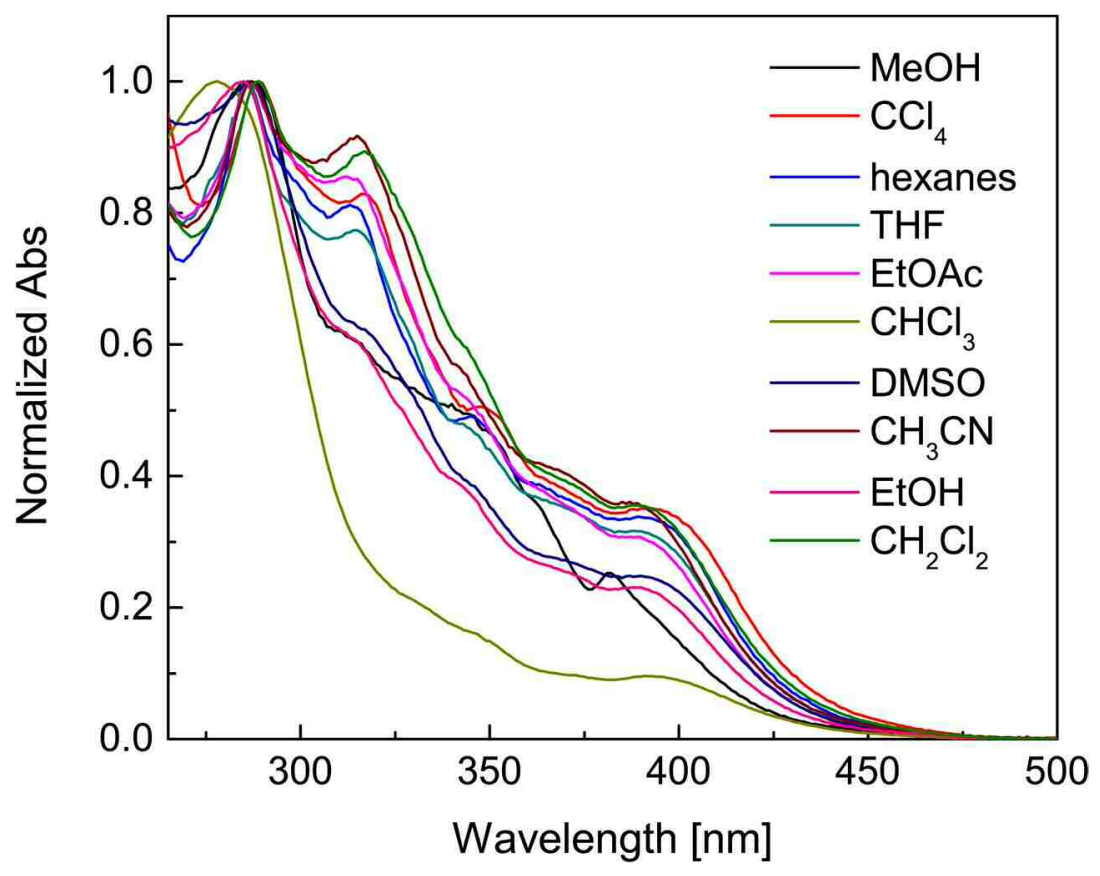

Fig. S-10 UV-Vis absorption spectra of 2 c $\left(8.98 \times 10^{-6} \mathrm{M}\right)$ measured in different organic solvents (all spectra are normalized). 


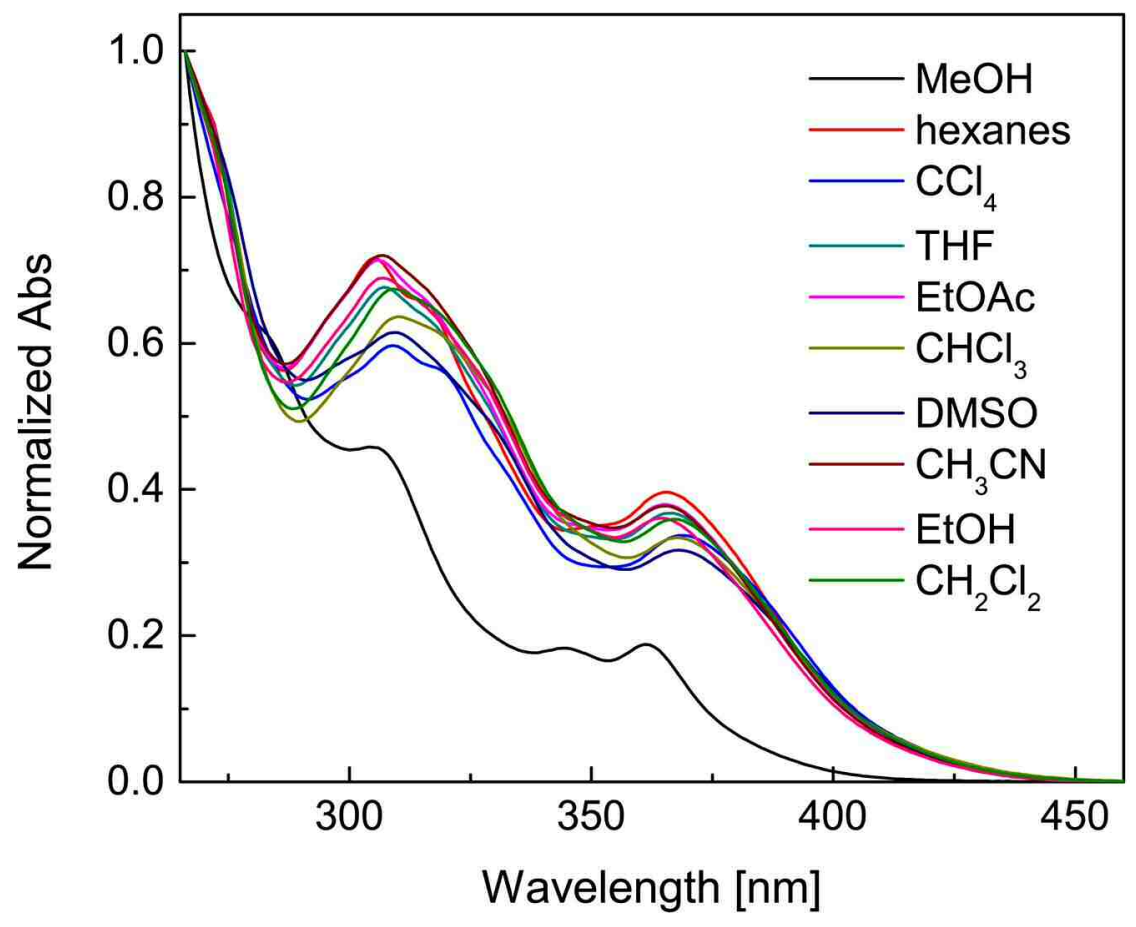

Fig. S-11 UV-Vis absorption spectra of $5\left(1.87 \times 10^{-5} \mathrm{M}\right)$ measured in different organic solvents (all spectra are normalized). 
Table S-1 Summary of UV-Vis Absorption and Fluorescence Data of Compound 2b

\begin{tabular}{|c|c|c|}
\hline Solvent & $\begin{array}{c}\text { UV-Vis absorption } \\
\lambda_{\max } / \mathrm{nm}\left(\varepsilon / \mathrm{mol}^{-1} \mathrm{~L} \mathrm{~cm}^{-1}\right)\end{array}$ & $\begin{array}{l}\text { Fluorescence } \\
\lambda_{\text {em }} / \mathrm{nm}\end{array}$ \\
\hline EtOH & $\begin{array}{l}395\left(6.98 \times 10^{3}\right), 345\left(\mathrm{sh}, 1.32 \times 10^{4}\right), 316\left(1.75 \times 10^{4}\right), 288 \\
\left(2.03 \times 10^{4}\right)\end{array}$ & $* *$ \\
\hline $\mathrm{CH}_{3} \mathrm{CN}$ & $394\left(4.97 \times 10^{3}\right), 317\left(1.25 \times 10^{4}\right), 288\left(1.38 \times 10^{4}\right)$ & 551 \\
\hline DMSO & $394\left(9.861 \times 10^{3}\right), 320\left(2.33 \times 10^{4}\right), 290\left(2.72 \times 10^{4}\right)$ & 553 \\
\hline Acetone & $395\left(1.42 \times 10^{4}\right), 372\left(\operatorname{sh}, 1.66 \times 10^{4}\right), 345\left(2.57 \times 10^{4}\right)$ & 544 \\
\hline $\mathrm{CHCl}_{3}$ & $\begin{array}{l}401\left(1.37 \times 10^{4}\right), 349\left(3.01 \times 10^{4}\right), 334\left(3.42 \times 10^{4}\right), 278 \\
\left(4.64 \times 10^{4}\right)\end{array}$ & 551 \\
\hline EtOAc & $\begin{array}{l}393\left(2.07 \times 10^{4}\right), 345\left(\mathrm{sh}, 3.58 \times 10^{4}\right), 316\left(5.10 \times 10^{4}\right), 288 \\
\left(5.88 \times 10^{4}\right)\end{array}$ & 524 \\
\hline THF & $\begin{array}{l}395\left(6.98 \times 10^{3}\right), 348\left(\mathrm{sh}, 3.10 \times 10^{4}\right), 318\left(4.42 \times 10^{4}\right), 289 \\
\left(5.19 \times 10^{4}\right)\end{array}$ & 523 \\
\hline Benzene & $\begin{array}{l}395\left(1.87 \times 10^{4}\right), 349\left(3.17 \times 10^{4}\right), 318\left(4.31 \times 10^{4}\right), 289 \\
\left(5.54 \times 10^{4}\right)\end{array}$ & 524 \\
\hline$p$-Xylene & $396\left(1.98 \times 10^{4}\right), 348\left(\mathrm{sh}, 3.37 \times 10^{4}\right), 318\left(4.52 \times 10^{4}\right)$ & 521 \\
\hline $\mathrm{CCl}_{4}$ & $\begin{array}{l}401\left(1.65 \times 10^{4}\right), 353\left(2.66 \times 10^{4}\right), 319\left(3.87 \times 10^{4}\right), 290 \\
\left(4.78 \times 10^{4}\right)\end{array}$ & 520 \\
\hline Hexane & $\begin{array}{l}399\left(1.96 \times 10^{4}\right), 348\left(3.29 \times 10^{4}\right), 316\left(4.76 \times 10^{4}\right), 287 \\
\left(5.86 \times 10^{4}\right)\end{array}$ & 495,514 \\
\hline $\mathrm{MeOH}$ & $\begin{array}{l}382\left(3.18 \times 10^{3}\right), 347\left(7.11 \times 10^{3}\right), 291\left(1.38 \times 10^{4}\right), 244 \\
\left(2.76 \times 10^{4}\right)\end{array}$ & ** \\
\hline
\end{tabular}

** Too weak to be determined accurately 
Table S-2 Summary of UV-Vis Absorption Data of Compound 2c

\begin{tabular}{ll}
\hline Solvent & \multicolumn{1}{c}{$\begin{array}{c}\text { UV-Vis absorption } \\
\lambda \max / \mathrm{nm}\left(\varepsilon / \mathrm{mol}^{-1} \mathrm{~L} \mathrm{~cm}^{-1}\right)\end{array}$} \\
\hline EtOH & $390\left(2.61 \times 10^{3}\right), 284\left(1.12 \times 10^{4}\right)$ \\
$\mathrm{CH}_{3} \mathrm{CN}$ & $389\left(3.02 \times 10^{3}\right), 315\left(7.34 \times 10^{3}\right), 285\left(8.03 \times 10^{3}\right)$ \\
DMSO & $391\left(2.76 \times 10^{3}\right), 345\left(\mathrm{sh}, 4.46 \times 10^{3}\right), 316\left(\mathrm{sh}, 7.16 \times 10^{3}\right), 285\left(1.16 \times 10^{4}\right)$ \\
Acetone & $391\left(2.23 \times 10^{3}\right)$ \\
$\mathrm{CHCl}_{3}$ & $395\left(2.26 \times 10^{3}\right), 278\left(2.44 \times 10^{4}\right)$ \\
EtOAc & $390\left(6.74 \times 10^{3}\right), 343\left(\mathrm{sh}, 8.63 \times 10^{3}\right), 313\left(1.16 \times 10^{4}\right), 286\left(1.30 \times 10^{4}\right)$ \\
THF & $395\left(2.64 \times 10^{3}\right), 345\left(\mathrm{sh}, 4.17 \times 10^{3}\right), 314\left(6.81 \times 10^{3}\right), 286\left(8.76 \times 10^{3}\right)$ \\
Benzene & $395\left(2.68 \times 10^{3}\right), 348\left(\mathrm{sh}, 4.31 \times 10^{3}\right), 284\left(2.15 \times 10^{4}\right)$ \\
$p$-Xylene & $392\left(2.78 \times 10^{3}\right), 347\left(\mathrm{sh}, 4.50 \times 10^{3}\right), 316\left(7.54 \times 10^{3}\right)$ \\
$\mathrm{CCl}_{4}$ & $395\left(2.75 \times 10^{3}\right), 350\left(\mathrm{sh}, 4.03 \times 10^{4}\right), 316\left(6.50 \times 10^{3}\right), 288\left(8.00 \times 10^{4}\right)$ \\
Hexane & $393\left(2.73 \times 10^{3}\right), 346\left(\mathrm{sh}, 3.93 \times 10^{3}\right), 313\left(6.44 \times 10^{3}\right), 283\left(7.80 \times 10^{3}\right)$ \\
MeOH & $382\left(2.12 \times 10^{3}\right), 346\left(\mathrm{sh}, 4.19 \times 10^{3}\right), 285\left(8.29 \times 10^{3}\right)$ \\
\hline
\end{tabular}

Table S-3 Summary of UV-Vis Absorption Data of Compound 5

\begin{tabular}{ll}
\hline Solvent & \multicolumn{1}{c}{$\begin{array}{c}\text { UV-Vis absorption } \\
\lambda \max / \mathrm{nm}\left(\varepsilon / \mathrm{mol}^{-1} \mathrm{~L} \mathrm{~cm}^{-1}\right)\end{array}$} \\
\hline EtOH & $366\left(1.19 \times 10^{4}\right), 307\left(2.27 \times 10^{4}\right), 251\left(5.63 \times 10^{4}\right)$ \\
$\mathrm{CH}_{3} \mathrm{CN}$ & $365\left(1.32 \times 10^{4}\right), 305\left(2.47 \times 10^{4}\right), 249\left(5.82 \times 10^{4}\right)$ \\
DMSO & $368\left(1.27 \times 10^{4}\right), 308\left(2.46 \times 10^{4}\right), 255\left(5.34 \times 10^{4}\right)$ \\
Acetone & $365\left(2.49 \times 10^{4}\right)$ \\
$\mathrm{CHCl}_{3}$ & $368\left(1.26 \times 10^{4}\right), 310\left(2.40 \times 10^{4}\right), 252\left(5.67 \times 10^{4}\right)$ \\
EtOAc & $366\left(1.57 \times 10^{4}\right), 306\left(2.81 \times 10^{4}\right), 250\left(6.43 \times 10^{4}\right)$ \\
THF & $354\left(1.65 \times 10^{4}\right), 297\left(2.70 \times 10^{4}\right), 245\left(6.02 \times 10^{4}\right)$ \\
Benzene & $369\left(1.28 \times 10^{4}\right), 308\left(2.29 \times 10^{4}\right), 274\left(3.78 \times 10^{4}\right)$ \\
$p$-Xylene & $368\left(1.27 \times 10^{4}\right), 319\left(2.13 \times 10^{4}\right), 308\left(2.30 \times 10^{4}\right)$ \\
CCl $_{4}$ & $369\left(1.26 \times 10^{4}\right), 318\left(\mathrm{sh}, 2.13 \times 10^{4}\right), 308\left(2.24 \times 10^{4}\right), 255\left(5.13 \times 10^{4}\right)$ \\
Hexane & $367\left(1.41 \times 10^{4}\right), 315\left(\mathrm{sh}, 2.37 \times 10^{4}\right), 305\left(2.58 \times 10^{4}\right), 249\left(6.53 \times 10^{4}\right)$ \\
MeOH & $362\left(8.17 \times 10^{3}\right), 305\left(2.04 \times 10^{4}\right), 282\left(\mathrm{sh} 2.75 \times 10^{4}\right), 258\left(6.25 \times 10^{4}\right)$ \\
\hline
\end{tabular}




\section{Photographic Images}
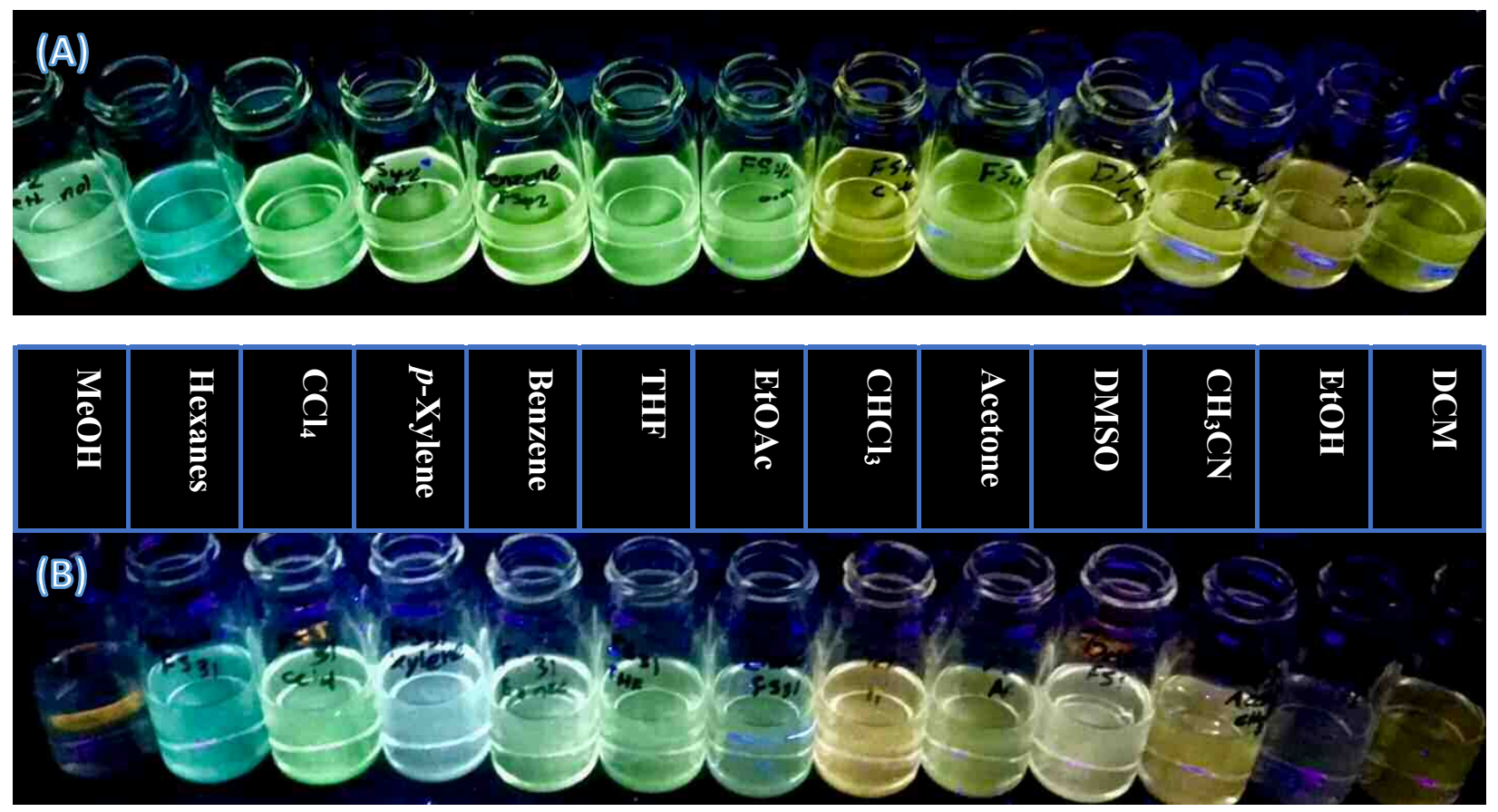

Fig. S-12 (A) Photographic images of $5\left(1.87 \times 10^{-5} \mathrm{M}\right)$ and $(\mathrm{B})$ Photographic images of 2c $(8.98$ $\left.\times 10^{-6} \mathrm{M}\right)$ dissolved or suspended in different organic solvents. 


\section{Cyclic Voltammetric Data of 2b}

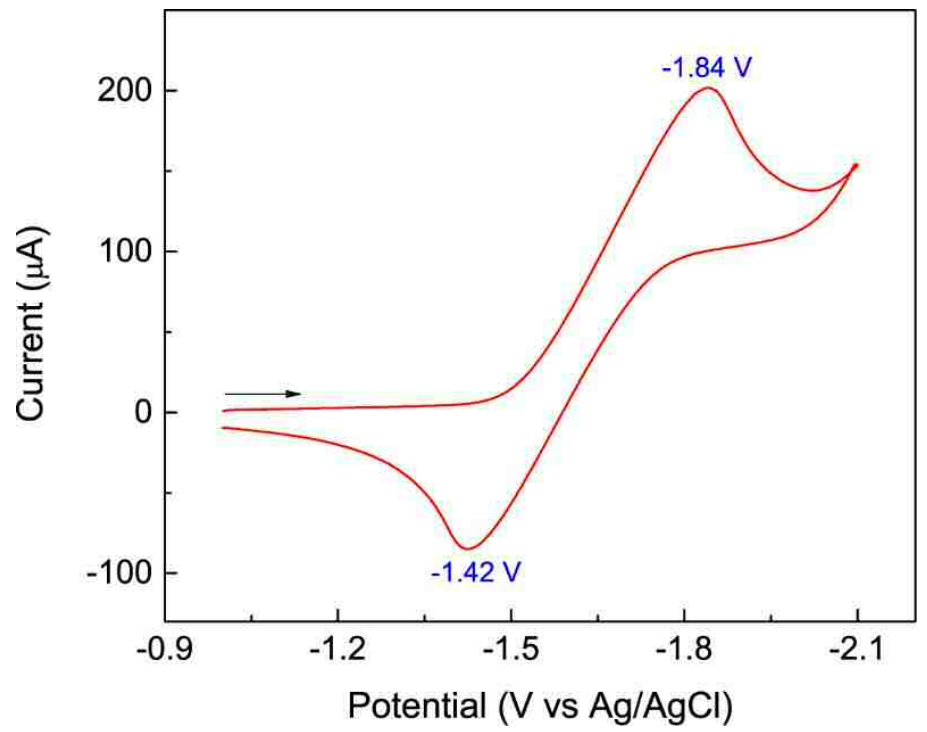

Fig. S-13 Cyclic voltammogram of $\mathbf{2 b}$ measured in $\mathrm{CH}_{2} \mathrm{Cl}_{2}$ at room temperature. Experimental conditions: $\mathrm{Bu}_{4} \mathrm{NBF}_{4}$ as electrolyte, glassy carbon as working electrode, $\mathrm{Pt}$ wire as counter electrode, $\mathrm{Ag} / \mathrm{AgCl}$ as reference electrode, scan rate $=200 \mathrm{mV} / \mathrm{s}$. 


\section{Detailed Results of DFT Calculations}
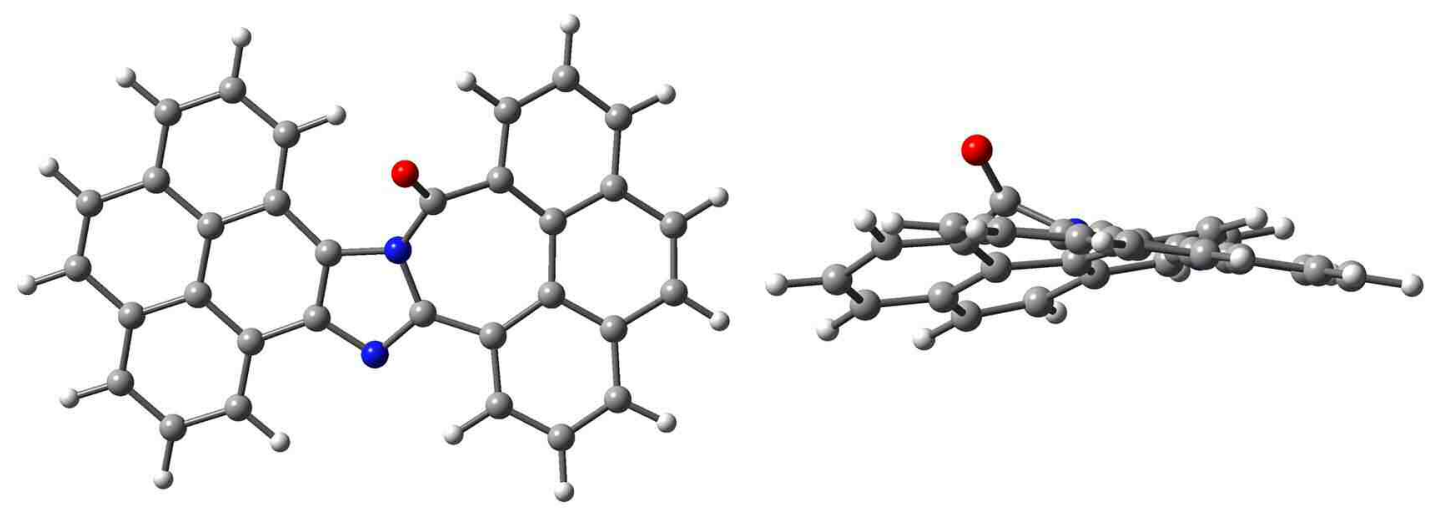

Fig. S-14 Optimized geometry of compound 2a calculated at the M06-2X/6-31G(d) level (top view and side view).
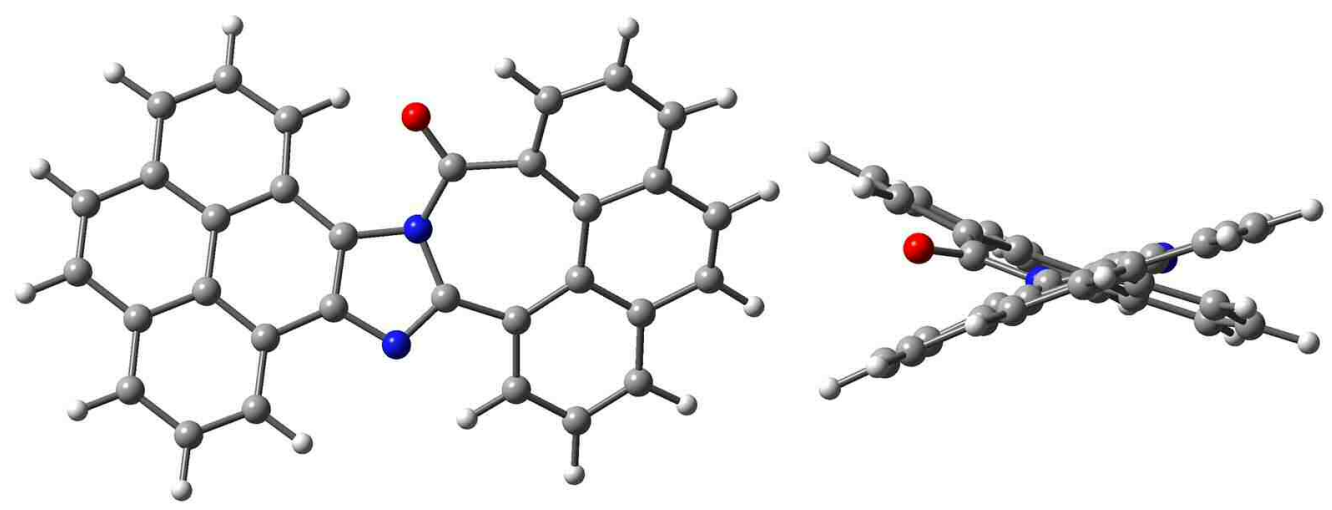

Fig. S-15 Transition state of compound 2a calculated at the M06-2X/6-31G(d) level (top view and side view). Imaginary frequency $=i 21.63 \mathrm{~cm}^{-1}$.

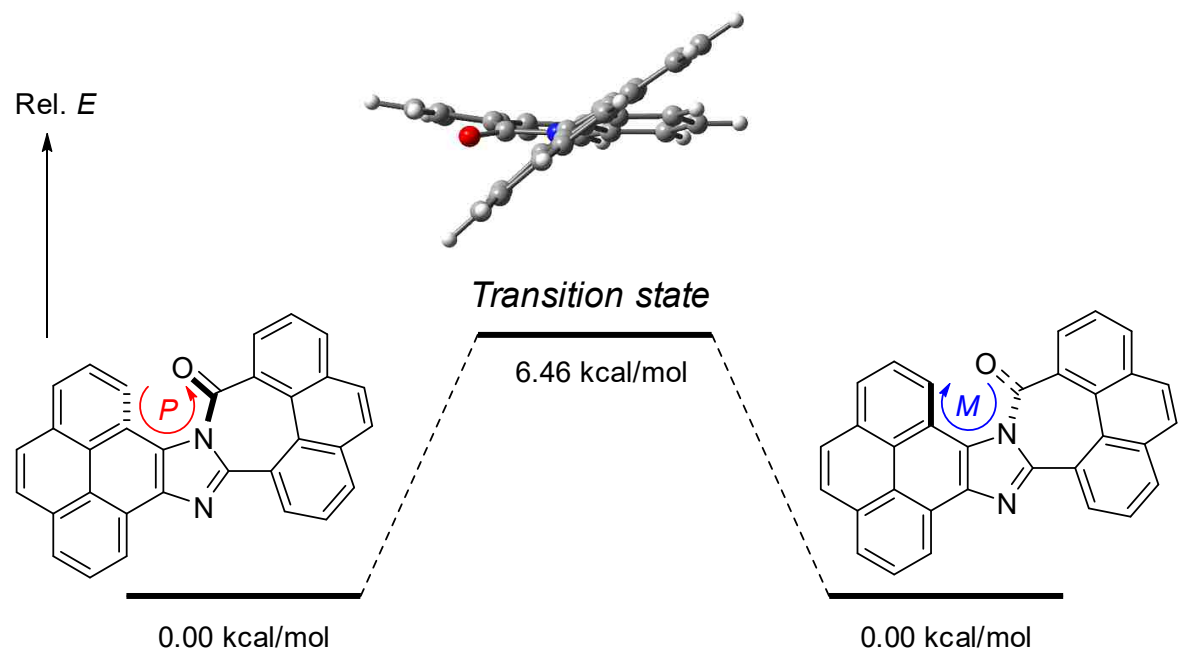

Fig. S-16 Energy profile for the epimerization process of 2a calculated at the M06-2X/6-31G(d) level. 


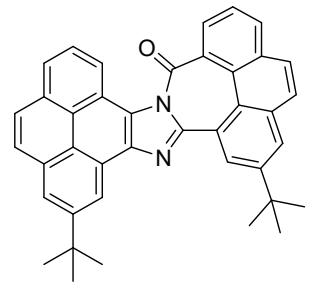

2c

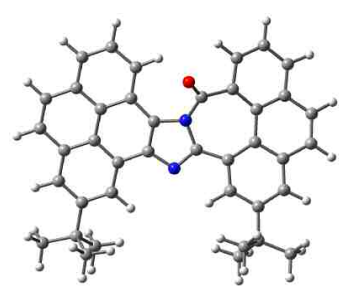

1.098

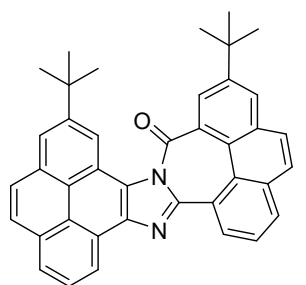

2d

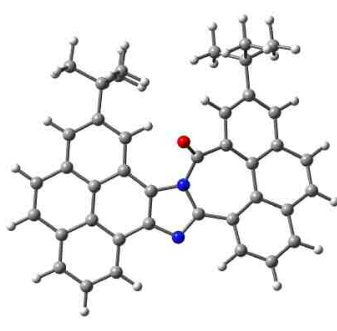

0.000
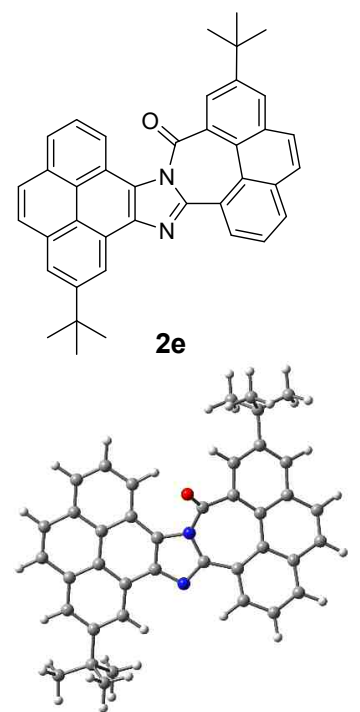

1.048

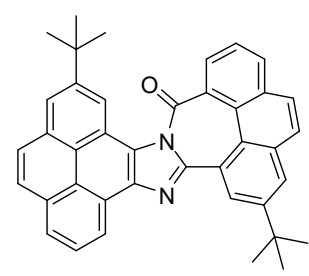

$2 f$

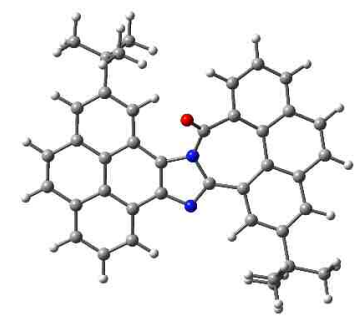

0.346

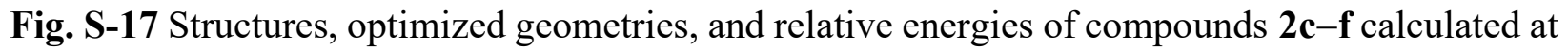
the M06-2X/6-31G(d) level.
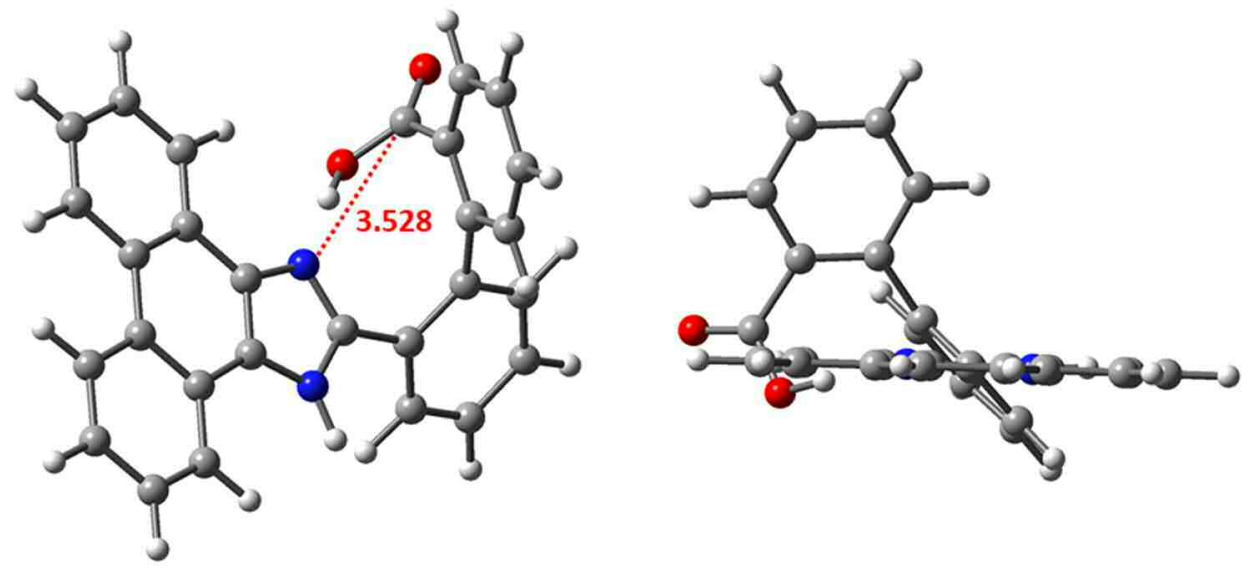

Fig. S-18 Optimized geometry of compound 4 calculated at the M06-2X/6-31G(d) level. The distance between imidazolyl $\mathrm{N}$ and carbonyl $\mathrm{C}$ atoms is indicated (in $\AA$ ). 


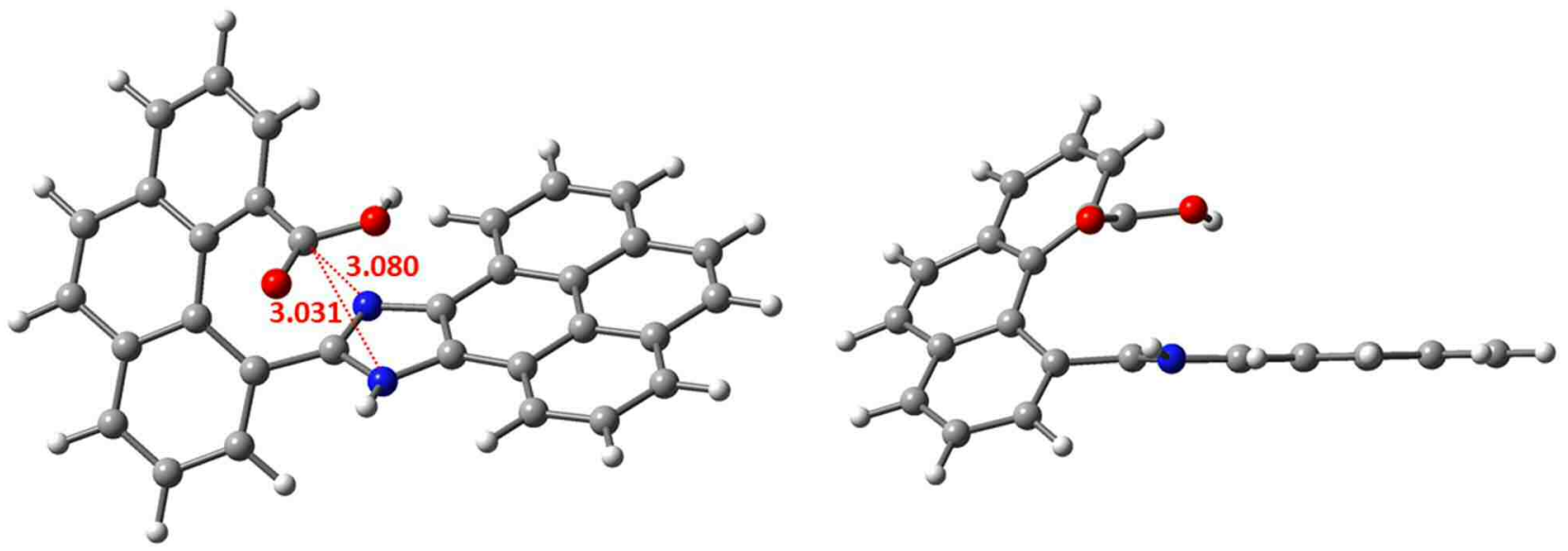

Fig. S-19 Optimized geometry of intermediate IM-5 calculated at the M06-2X/6-31G(d) level. The distances between imidazolyl $\mathrm{N}$ and carbonyl $\mathrm{C}$ atoms are indicated (in $\AA$ ).
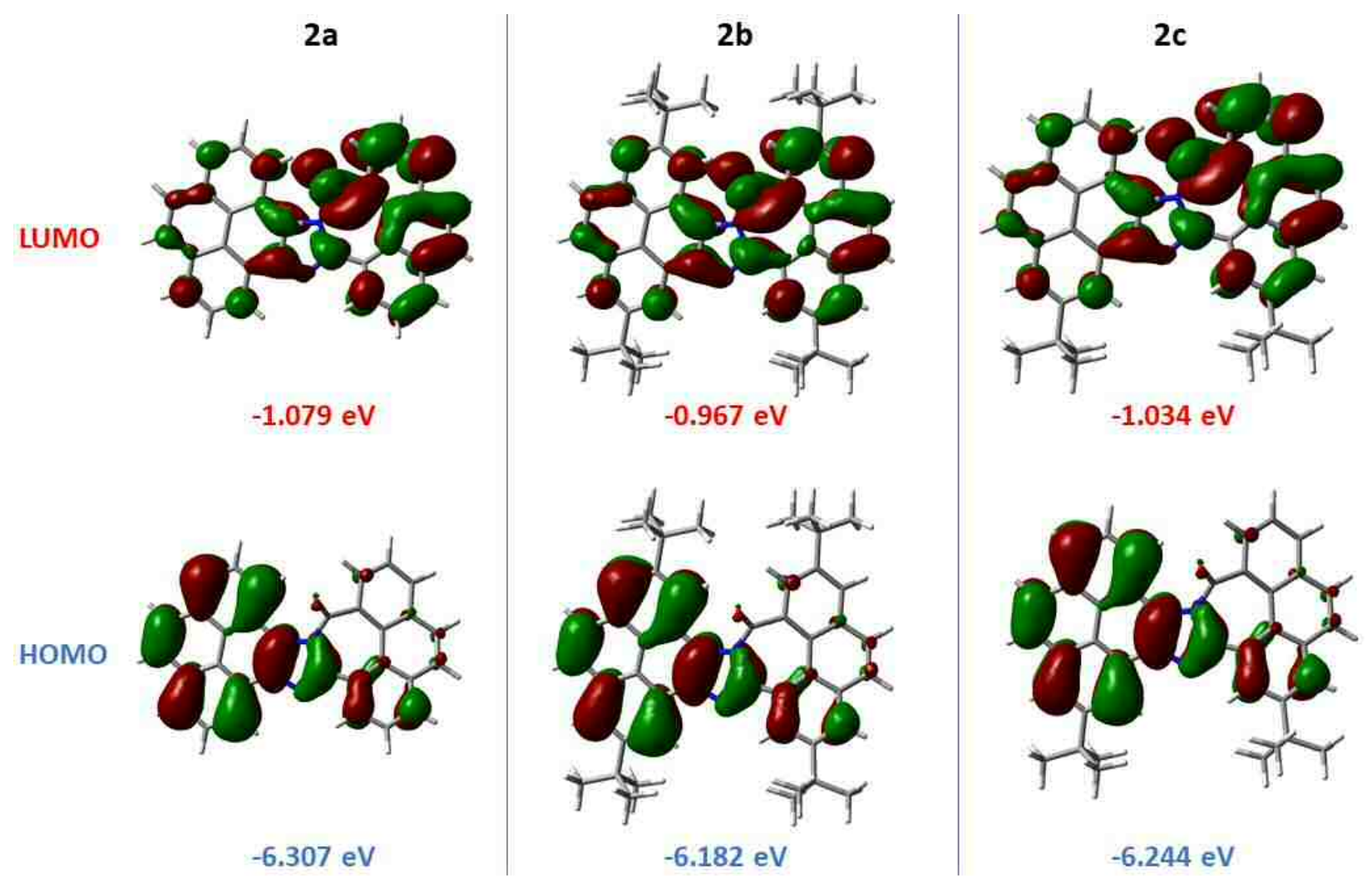

Fig. S-20 Plots and energies of frontier molecular orbitals for 2a-c calculated at the M06-2X/6$31 \mathrm{G}(\mathrm{d})$ level. 


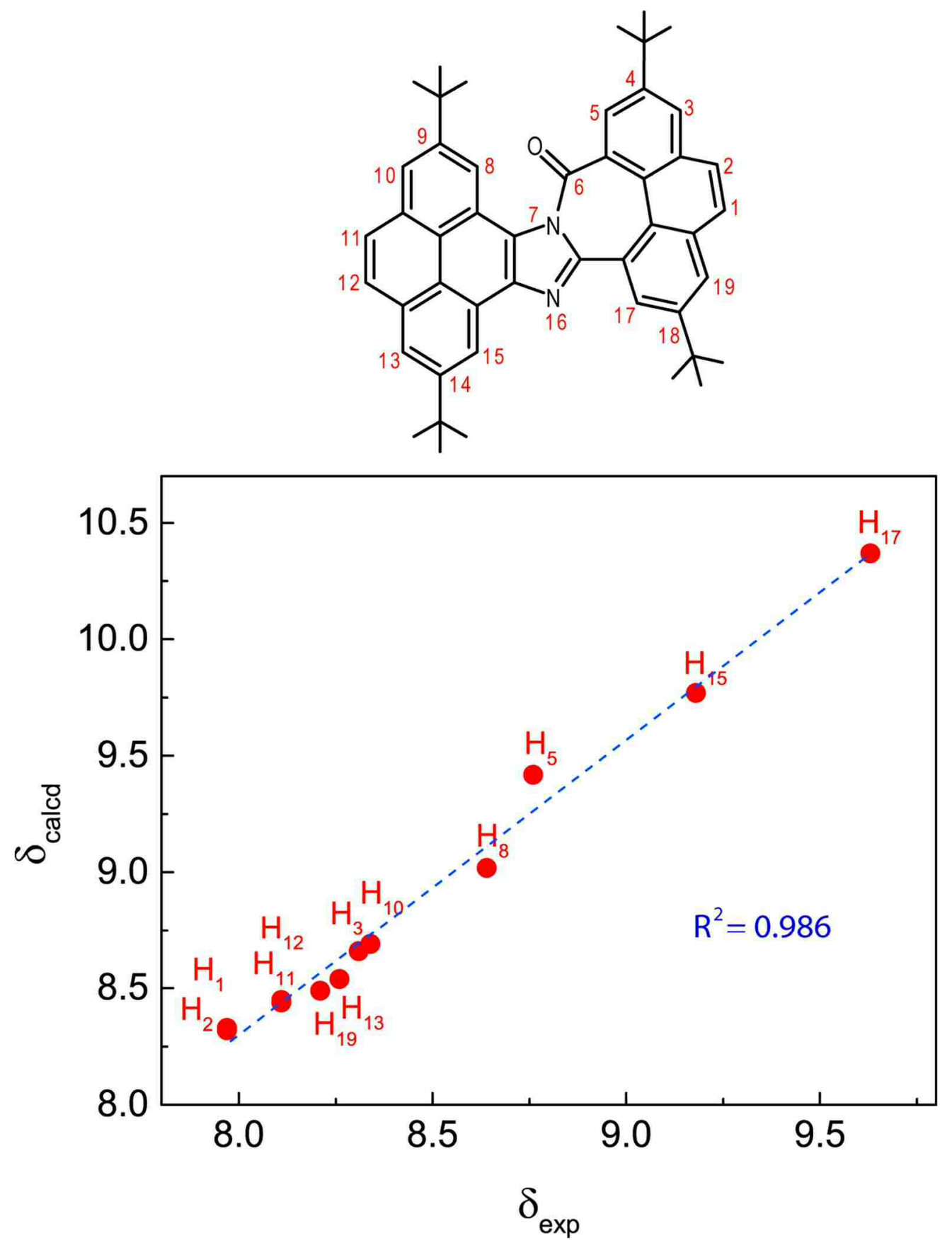

Fig. S-21 Correlation of DFT calculated and experimental observed chemical shifts for the aromatic protons in compound $\mathbf{2 b}$. 

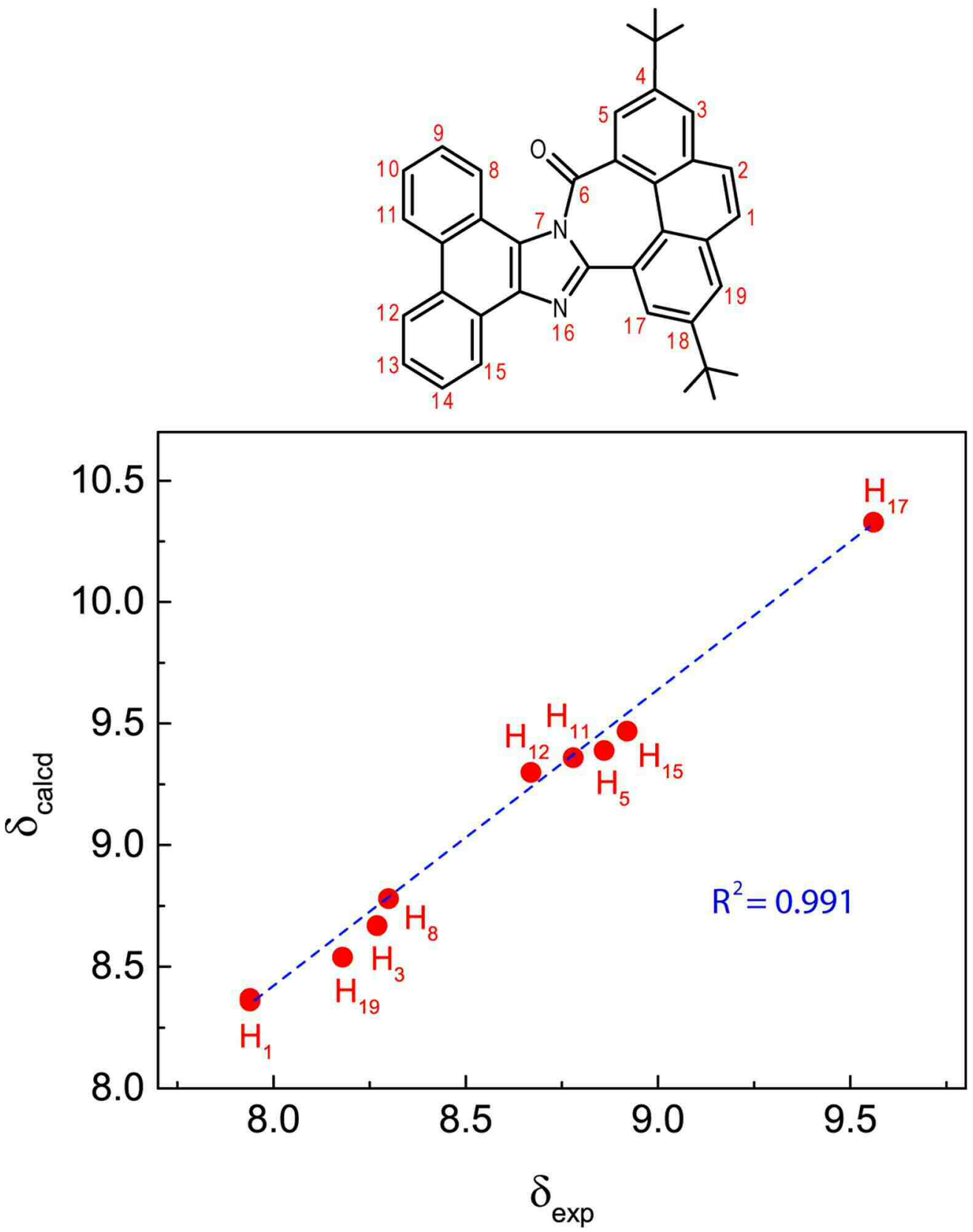

Fig. S-22 Correlation of DFT calculated and experimental observed chemical shifts for the aromatic protons in compound 5. Signals at positions 2, 14, 9, 13, and 10 are overlapped in the experimental spectrum and they are therefore not shown in this correlation diagram. 
Table S-4 Cartesian Coordinates and Energies of Optimized Geometries

\begin{tabular}{|c|c|c|c|c|c|c|c|}
\hline \multicolumn{4}{|c|}{ 2a $(E=-1413.315363$ Hartree $)$} & \multicolumn{4}{|c|}{ Transition state $\mathbf{2 a}(E=-1413.307260$ Hartree $)$} \\
\hline $\mathrm{C}$ & 2.83194700 & -3.50576300 & 0.09485500 & $\mathrm{H}$ & -2.81197600 & -4.19671300 & 1.90570300 \\
\hline $\mathrm{C}$ & 1.78577200 & -2.59044800 & 0.17477700 & $\mathrm{C}$ & -2.99134700 & -3.23927500 & 1.42695000 \\
\hline $\mathrm{C}$ & 2.03335300 & -1.21532400 & 0.14765600 & $\mathrm{C}$ & -3.43490800 & -0.73061800 & 0.25457300 \\
\hline $\mathrm{C}$ & 3.38042800 & -0.75470300 & -0.01256800 & $\mathrm{C}$ & -1.90819100 & -2.42916400 & 1.10313300 \\
\hline $\mathrm{C}$ & 4.43517600 & -1.70554900 & -0.07652800 & $\mathrm{C}$ & -4.28655300 & -2.84055800 & 1.13713000 \\
\hline $\mathrm{C}$ & 4.14300100 & -3.07358200 & -0.01388300 & $\mathrm{C}$ & -4.52746600 & -1.58786400 & 0.56331700 \\
\hline $\mathrm{C}$ & 1.03434300 & -0.17539500 & 0.22228000 & $\mathrm{C}$ & -2.09602700 & -1.19171600 & 0.47773400 \\
\hline $\mathrm{C}$ & 3.69796400 & 0.64248800 & -0.14186300 & $\mathrm{H}$ & -5.12929800 & -3.48525000 & 1.37156300 \\
\hline $\mathrm{C}$ & 2.67713100 & 1.62824200 & -0.13940900 & $\mathrm{C}$ & -3.71596200 & 0.59174000 & -0.23376600 \\
\hline $\mathrm{C}$ & 1.33049800 & 1.15939000 & 0.03808500 & $\mathrm{C}$ & -4.25781200 & 3.20671400 & -1.11838200 \\
\hline $\mathrm{C}$ & 2.98895500 & 2.97701100 & -0.29422800 & $\mathrm{C}$ & -2.66755500 & 1.51937200 & -0.45921400 \\
\hline $\mathrm{H}$ & 2.18199200 & 3.70249100 & -0.29225400 & $\mathrm{C}$ & -5.05558400 & 1.01118600 & -0.45461300 \\
\hline $\mathrm{C}$ & 4.31637600 & 3.37088800 & -0.44349600 & $\mathrm{C}$ & -5.30120900 & 2.31535500 & -0.90372800 \\
\hline $\mathrm{C}$ & 5.33206100 & 2.42457500 & -0.44554500 & $\mathrm{C}$ & -2.94122900 & 2.81582100 & -0.89137100 \\
\hline $\mathrm{C}$ & 5.04655300 & 1.05993100 & -0.30186300 & $\mathrm{H}$ & -6.32878000 & 2.62527400 & -1.07434300 \\
\hline $\mathrm{C}$ & 6.08715100 & 0.06743500 & -0.32441000 & $\mathrm{H}$ & -2.11385200 & 3.49926800 & -1.05206200 \\
\hline $\mathrm{C}$ & 5.79306000 & -1.24855200 & -0.22113200 & $\mathrm{H}$ & -4.47009300 & 4.21411700 & -1.46245100 \\
\hline $\mathrm{H}$ & 6.58022100 & -1.99714000 & -0.25116100 & $\mathrm{C}$ & -5.87270800 & -1.14403800 & 0.30218800 \\
\hline $\mathrm{H}$ & 7.11513300 & 0.40103200 & -0.43774100 & $\mathrm{H}$ & -6.68481000 & -1.83179000 & 0.52252500 \\
\hline $\mathrm{H}$ & 0.77455700 & -2.96101900 & 0.26474100 & $\mathrm{C}$ & -6.12745400 & 0.08914800 & -0.19053200 \\
\hline $\mathrm{H}$ & 4.95816700 & -3.79018500 & -0.06448700 & $\mathrm{H}$ & -7.14692100 & 0.41523200 & -0.37791800 \\
\hline $\mathrm{H}$ & 6.36725600 & 2.73297100 & -0.56517000 & $\mathrm{C}$ & -1.05254400 & -0.25594700 & 0.11935300 \\
\hline $\mathrm{N}$ & 0.19993900 & 1.93110200 & 0.04553500 & $\mathrm{C}$ & -1.33255800 & 1.04371800 & -0.23229000 \\
\hline $\mathrm{C}$ & -0.80092100 & 1.10274000 & 0.21566500 & $\mathrm{~N}$ & 0.36284000 & -0.31624800 & 0.15767600 \\
\hline $\mathrm{N}$ & -0.35858800 & -0.20955000 & 0.32283700 & $\mathrm{~N}$ & -0.19004400 & 1.80065700 & -0.32516100 \\
\hline $\mathrm{C}$ & -1.12575800 & -1.26688000 & 0.87191000 & $\mathrm{C}$ & 0.80363100 & 0.99813900 & -0.06643000 \\
\hline $\mathrm{C}$ & -2.44704000 & -1.55450300 & 0.24281100 & $\mathrm{C}$ & 1.04923800 & -1.47946800 & -0.24828000 \\
\hline $\mathrm{C}$ & -3.44754700 & -0.61710800 & -0.14287400 & $\mathrm{C}$ & 2.52899800 & -1.50419700 & -0.53332900 \\
\hline $\mathrm{C}$ & -2.18873200 & 1.57795000 & 0.33118600 & $\mathrm{C}$ & 5.18893900 & -2.27344800 & -0.95280200 \\
\hline $\mathrm{C}$ & -3.37178900 & 0.83160200 & 0.04264300 & $\mathrm{C}$ & 2.86603400 & -2.72141500 & -1.12672500 \\
\hline $\mathrm{O}$ & -0.63989500 & -1.98406900 & 1.71067400 & $\mathrm{C}$ & 3.55676300 & -0.56319200 & -0.20391100 \\
\hline $\mathrm{C}$ & -3.79940600 & -3.43542600 & -0.49744900 & $\mathrm{C}$ & 4.90054100 & -1.02603000 & -0.37146400 \\
\hline $\mathrm{C}$ & -4.79934400 & -2.56043500 & -0.84820800 & $\mathrm{C}$ & 4.18121200 & -3.10600300 & -1.36993400 \\
\hline $\mathrm{C}$ & -4.66245600 & -1.17438400 & -0.64089100 & $\mathrm{H}$ & 4.39395200 & -4.05925000 & -1.84143900 \\
\hline $\mathrm{C}$ & -2.64130900 & -2.92253100 & 0.08740800 & $\mathrm{H}$ & 6.23141400 & -2.55706900 & -1.06842300 \\
\hline $\mathrm{H}$ & -5.73444000 & -2.92664300 & -1.26295900 & $\mathrm{C}$ & 3.37516800 & 0.81481600 & 0.25187200 \\
\hline $\mathrm{H}$ & -1.86443800 & -3.59260600 & 0.43784400 & $\mathrm{C}$ & 3.23680900 & 3.55769400 & 1.04618500 \\
\hline $\mathrm{C}$ & -5.78577700 & 0.96029200 & -0.55252100 & $\mathrm{C}$ & 4.53140300 & 1.55772400 & 0.64494600 \\
\hline $\mathrm{H}$ & -6.66764100 & 1.58386300 & -0.66720300 & $\mathrm{C}$ & 2.13975800 & 1.52423400 & 0.25701800 \\
\hline $\mathrm{C}$ & -4.58055200 & 1.57959400 & -0.08015500 & $\mathrm{C}$ & 2.09944900 & 2.86595700 & 0.63899200 \\
\hline $\mathrm{C}$ & -5.81077000 & -0.34739600 & -0.87470200 & $\mathrm{C}$ & 4.44219300 & 2.89927400 & 1.05594000 \\
\hline $\mathrm{H}$ & -6.71096400 & -0.81600300 & -1.26127900 & $\mathrm{H}$ & 1.13850600 & 3.36304400 & 0.62334300 \\
\hline $\mathrm{C}$ & -2.27431800 & 2.93809600 & 0.62355200 & $\mathrm{H}$ & 5.35356700 & 3.40668400 & 1.35922000 \\
\hline $\mathrm{C}$ & -4.62267000 & 2.95441300 & 0.21347400 & $\mathrm{H}$ & 3.16185600 & 4.59636800 & 1.35026700 \\
\hline $\mathrm{C}$ & -3.48566900 & 3.62595300 & 0.59650700 & $\mathrm{C}$ & 5.84277100 & 0.98461500 & 0.57853700 \\
\hline $\mathrm{H}$ & -1.35285000 & 3.46338900 & 0.84129800 & $\mathrm{H}$ & 6.68323900 & 1.58519600 & 0.91346300 \\
\hline $\mathrm{H}$ & -5.57329100 & 3.47260800 & 0.12424200 & $\mathrm{C}$ & 6.02216300 & -0.24109500 & 0.05506900 \\
\hline $\mathrm{H}$ & -3.91439800 & -4.50455200 & -0.63927200 & $\mathrm{H}$ & 7.01433900 & -0.66898200 & -0.05363000 \\
\hline $\mathrm{H}$ & 2.61074600 & -4.56798100 & 0.12365300 & $\mathrm{H}$ & -0.90827600 & -2.76864700 & 1.32936400 \\
\hline $\mathrm{H}$ & 4.55739400 & 4.42286400 & -0.55989800 & $\mathrm{H}$ & 2.05890000 & -3.39764100 & -1.37311700 \\
\hline $\mathrm{H}$ & -3.51598400 & 4.68289200 & 0.83914100 & $\mathrm{O}$ & 0.39661000 & -2.48744700 & -0.40458300 \\
\hline
\end{tabular}




\begin{tabular}{|c|c|c|c|c|c|c|c|}
\hline \multicolumn{4}{|c|}{ 2b $(E=-2042.029809$ Hartree $)$} & \multicolumn{4}{|c|}{ 2c $(E=-1727.671879$ Hartree $)$} \\
\hline $\mathrm{C}$ & -0.16743400 & -4.49711300 & 0.31219200 & $\mathrm{C}$ & 2.34048500 & -4.88665400 & 0.30505600 \\
\hline $\mathrm{C}$ & -0.32210900 & -3.10962500 & 0.30264700 & $\mathrm{C}$ & 1.35983500 & -3.89690800 & 0.30926400 \\
\hline $\mathrm{C}$ & 0.77063700 & -2.24134800 & 0.24373100 & $\mathrm{C}$ & 1.70757700 & -2.54468900 & 0.26320800 \\
\hline $\mathrm{C}$ & 2.08514000 & -2.77998500 & 0.12050700 & $\mathrm{C}$ & 3.09125000 & -2.18752700 & 0.16315000 \\
\hline $\mathrm{C}$ & 2.24697400 & -4.19066200 & 0.13429300 & $\mathrm{C}$ & 4.07701900 & -3.21091900 & 0.17660700 \\
\hline $\mathrm{C}$ & 1.12423700 & -5.01718200 & 0.24306400 & $\mathrm{C}$ & 3.68318800 & -4.55302000 & 0.25530800 \\
\hline $\mathrm{C}$ & 0.69807400 & -0.80042100 & 0.25379300 & $\mathrm{C}$ & 0.78651900 & -1.43103300 & 0.26074100 \\
\hline $\mathrm{C}$ & 3.23849600 & -1.94239400 & -0.04891200 & $\mathrm{C}$ & 3.51543100 & -0.82226100 & 0.01805700 \\
\hline $\mathrm{C}$ & 3.11924100 & -0.53047800 & -0.12112900 & $\mathrm{C}$ & 2.57570200 & 0.23737900 & -0.05746400 \\
\hline $\mathrm{C}$ & 1.79374400 & 0.00783000 & 0.03097100 & $\mathrm{C}$ & 1.18970700 & -0.12707300 & 0.06023500 \\
\hline $\mathrm{C}$ & 4.24166900 & 0.26532400 & -0.31648300 & $\mathrm{C}$ & 2.99615900 & 1.55030300 & -0.22811000 \\
\hline $\mathrm{H}$ & 4.09446100 & 1.34053000 & -0.36849800 & $\mathrm{H}$ & 2.23219300 & 2.32068000 & -0.28476000 \\
\hline $\mathrm{C}$ & 5.52183900 & -0.29310300 & -0.43788100 & $\mathrm{C}$ & 4.35679000 & 1.87621300 & -0.32059700 \\
\hline $\mathrm{C}$ & 5.64016100 & -1.67907700 & -0.36311700 & $\mathrm{C}$ & 5.28175300 & 0.83881200 & -0.24301600 \\
\hline $\mathrm{C}$ & 4.52772000 & -2.51363800 & -0.17628600 & $\mathrm{C}$ & 4.89122200 & -0.49990000 & -0.08147800 \\
\hline $\mathrm{C}$ & 4.65915900 & -3.94606400 & -0.12130900 & $\mathrm{C}$ & 5.85937900 & -1.56156000 & -0.02617100 \\
\hline $\mathrm{C}$ & 3.57381000 & -4.74188700 & 0.02312600 & $\mathrm{C}$ & 5.46856000 & -2.85175600 & 0.09248200 \\
\hline $\mathrm{H}$ & 3.68023300 & -5.82313200 & 0.05199500 & $\mathrm{H}$ & 6.20236000 & -3.65285300 & 0.12118700 \\
\hline $\mathrm{H}$ & 5.65362600 & -4.37562000 & -0.20943900 & $\mathrm{H}$ & 6.91298200 & -1.30366400 & -0.09335300 \\
\hline $\mathrm{H}$ & -1.31568400 & -2.69288200 & 0.34792200 & $\mathrm{H}$ & 2.04132500 & -5.92919200 & 0.34674600 \\
\hline $\mathrm{H}$ & 1.28161900 & -6.09351500 & 0.26006200 & $\mathrm{H}$ & 0.32114400 & -4.19257200 & 0.35593400 \\
\hline $\mathrm{H}$ & 6.61256100 & -2.15286900 & -0.45479900 & $\mathrm{H}$ & 4.44535400 & -5.32742400 & 0.26394900 \\
\hline $\mathrm{N}$ & 1.43846600 & 1.32951900 & -0.01770000 & $\mathrm{H}$ & 6.34526900 & 1.04477100 & -0.31289400 \\
\hline $\mathrm{C}$ & 0.13960100 & 1.36198800 & 0.15858100 & $\mathrm{~N}$ & 0.12068100 & 0.72477200 & -0.01086100 \\
\hline $\mathrm{N}$ & -0.37936300 & 0.08404800 & 0.32426500 & $\mathrm{C}$ & -0.94663900 & -0.02310100 & 0.12692100 \\
\hline $\mathrm{C}$ & -1.64445200 & -0.20424800 & 0.89751300 & $\mathrm{~N}$ & -0.60872300 & -1.36047300 & 0.28911400 \\
\hline $\mathrm{C}$ & -2.84305900 & 0.40022000 & 0.25163600 & $\mathrm{C}$ & -1.47622100 & -2.34562500 & 0.82447300 \\
\hline $\mathrm{C}$ & -2.99914600 & 1.75066500 & -0.17296500 & $\mathrm{C}$ & -2.78451300 & -2.55709600 & 0.14090300 \\
\hline $\mathrm{C}$ & -0.60870400 & 2.62811900 & 0.22112700 & $\mathrm{C}$ & -3.69205400 & -1.56161200 & -0.32267000 \\
\hline $\mathrm{C}$ & -2.00056400 & 2.80708100 & -0.04972500 & $\mathrm{C}$ & -2.30064500 & 0.55326000 & 0.15370100 \\
\hline $\mathrm{O}$ & -1.72750900 & -1.02516400 & 1.77748500 & $\mathrm{C}$ & -3.51621200 & -0.11921700 & -0.17892700 \\
\hline $\mathrm{C}$ & -5.12076400 & -0.20263500 & -0.43293400 & $\mathrm{O}$ & -1.08214900 & -3.07119900 & 1.70345800 \\
\hline $\mathrm{C}$ & -5.30257100 & 1.11221000 & -0.80371300 & $\mathrm{C}$ & -4.24172200 & -4.35601700 & -0.60465300 \\
\hline $\mathrm{C}$ & -4.29627600 & 2.08867700 & -0.65018800 & $\mathrm{C}$ & -5.15411700 & -3.42162100 & -1.03235900 \\
\hline $\mathrm{C}$ & -3.87531600 & -0.51877000 & 0.13519900 & $\mathrm{C}$ & -4.92121800 & -2.04303200 & -0.86338500 \\
\hline $\mathrm{H}$ & -6.25417100 & 1.44613900 & -1.20588200 & $\mathrm{C}$ & -3.07567500 & -3.91105900 & 0.01939900 \\
\hline $\mathrm{H}$ & -3.69293300 & -1.52163900 & 0.50994500 & $\mathrm{H}$ & -4.43154300 & -5.41786900 & -0.71779200 \\
\hline $\mathrm{C}$ & -3.76135900 & 4.44363500 & -0.67220900 & $\mathrm{H}$ & -6.09378100 & -3.73129100 & -1.48158000 \\
\hline $\mathrm{H}$ & -4.03159900 & 5.48457200 & -0.82521900 & $\mathrm{H}$ & -2.36920100 & -4.62473300 & 0.42799200 \\
\hline $\mathrm{C}$ & -2.43033800 & 4.15278000 & -0.22373900 & $\mathrm{C}$ & -5.87336200 & 0.17534600 & -0.90565800 \\
\hline $\mathrm{C}$ & -4.63992600 & 3.45271600 & -0.92624700 & $\mathrm{H}$ & -6.69643200 & 0.86016800 & -1.08851400 \\
\hline $\mathrm{H}$ & -5.63946400 & 3.66719200 & -1.29321100 & $\mathrm{C}$ & -4.64856700 & 0.71646100 & -0.39056700 \\
\hline $\mathrm{C}$ & 0.20649100 & 3.73228400 & 0.43974200 & $\mathrm{C}$ & -5.98691100 & -1.13869100 & -1.18304100 \\
\hline $\mathrm{C}$ & -1.56176800 & 5.24006100 & -0.00312000 & $\mathrm{H}$ & -6.90183300 & -1.54983700 & -1.59946800 \\
\hline $\mathrm{C}$ & -0.24520900 & 5.05819800 & 0.36243800 & $\mathrm{C}$ & -2.30481800 & 1.92150300 & 0.39822800 \\
\hline $\mathrm{H}$ & 1.25147100 & 3.52984000 & 0.64434800 & $\mathrm{C}$ & -4.60015700 & 2.10254400 & -0.14346900 \\
\hline $\mathrm{H}$ & -1.97082000 & 6.23675500 & -0.13638900 & $\mathrm{C}$ & -3.44843300 & 2.72605600 & 0.28669200 \\
\hline $\mathrm{C}$ & -1.36877800 & -5.44153500 & 0.41539400 & $\mathrm{H}$ & -1.35190900 & 2.37172200 & 0.65103600 \\
\hline $\mathrm{C}$ & -2.69883900 & -4.67897800 & 0.45300400 & $\mathrm{H}$ & -5.51207300 & 2.66794400 & -0.30809400 \\
\hline $\mathrm{H}$ & -2.75250300 & -4.00272600 & 1.31414500 & $\mathrm{C}$ & 4.76584800 & 3.34009100 & -0.50209000 \\
\hline $\mathrm{H}$ & -2.84246000 & -4.09118200 & -0.46099200 & $\mathrm{C}$ & -3.35439100 & 4.21908200 & 0.59609000 \\
\hline $\mathrm{H}$ & -3.52834500 & -5.38979100 & 0.52769700 & $\mathrm{C}$ & -4.70199100 & 4.92479200 & 0.41906300 \\
\hline $\mathrm{C}$ & -1.24888700 & -6.26870400 & 1.70628100 & $\mathrm{H}$ & -4.59474700 & 5.98569000 & 0.66594300 \\
\hline $\mathrm{H}$ & -1.23320600 & -5.61504100 & 2.58414200 & $\mathrm{H}$ & -5.05963900 & 4.85792100 & -0.61398000 \\
\hline $\mathrm{H}$ & -2.10057900 & -6.95223000 & 1.79823700 & $\mathrm{H}$ & -5.46694300 & 4.50265900 & 1.07960500 \\
\hline
\end{tabular}




\begin{tabular}{|c|c|c|c|c|c|c|c|}
\hline $\mathrm{H}$ & -0.33330100 & -6.86803800 & 1.71467800 & $\mathrm{C}$ & -2.89368500 & 4.40219800 & 2.05188400 \\
\hline $\mathrm{C}$ & -1.38751300 & -6.38566500 & -0.79795000 & $\mathrm{H}$ & -3.60712200 & 3.94631400 & 2.74601200 \\
\hline $\mathrm{H}$ & -1.46743900 & -5.81767500 & -1.73053500 & $\mathrm{H}$ & -1.91420900 & 3.94646400 & 2.22502900 \\
\hline $\mathrm{H}$ & -0.47888600 & -6.99301900 & -0.85008100 & $\mathrm{H}$ & -2.81579400 & 5.46891600 & 2.28910700 \\
\hline $\mathrm{H}$ & -2.24363200 & -7.06671200 & -0.73400700 & $\mathrm{C}$ & -2.33222700 & 4.87097000 & -0.35061100 \\
\hline $\mathrm{C}$ & 0.72708100 & 6.20395200 & 0.63787000 & $\mathrm{H}$ & -1.33763800 & 4.42890900 & -0.23918900 \\
\hline $\mathrm{C}$ & 0.04996200 & 7.57139200 & 0.50771300 & $\mathrm{H}$ & -2.63962800 & 4.75230400 & -1.39461700 \\
\hline $\mathrm{H}$ & 0.77485700 & 8.36132400 & 0.72808500 & $\mathrm{H}$ & -2.25203500 & 5.94202700 & -0.13435500 \\
\hline $\mathrm{H}$ & -0.32761000 & 7.73948800 & -0.50655400 & $\mathrm{C}$ & 4.24981800 & 4.16225100 & 0.69082900 \\
\hline $\mathrm{H}$ & -0.78366900 & 7.67585300 & 1.21043700 & $\mathrm{H}$ & 4.53248300 & 5.21458500 & 0.57396700 \\
\hline $\mathrm{C}$ & 1.27660700 & 6.06432500 & 2.06742100 & $\mathrm{H}$ & 3.15991300 & 4.11285300 & 0.77248100 \\
\hline $\mathrm{H}$ & 0.46615600 & 6.11169300 & 2.80197000 & $\mathrm{H}$ & 4.67587700 & 3.79296600 & 1.62931500 \\
\hline $\mathrm{H}$ & 1.80393900 & 5.11569900 & 2.20556300 & $\mathrm{C}$ & 6.28581300 & 3.51147700 & -0.58396900 \\
\hline $\mathrm{H}$ & 1.98150200 & 6.87585200 & 2.27920700 & $\mathrm{H}$ & 6.78023300 & 3.16384500 & 0.32929800 \\
\hline $\mathrm{C}$ & 1.89003700 & 6.13615800 & -0.36661700 & $\mathrm{H}$ & 6.70739400 & 2.96669400 & -1.43533300 \\
\hline $\mathrm{H}$ & 2.43548500 & 5.19071500 & -0.29131600 & $\mathrm{H}$ & 6.52783600 & 4.57137400 & -0.71243700 \\
\hline $\mathrm{H}$ & 1.52202500 & 6.23296800 & -1.39313600 & $\mathrm{C}$ & 4.14682500 & 3.88209400 & -1.80149400 \\
\hline $\mathrm{H}$ & 2.59756200 & 6.95063700 & -0.17564500 & $\mathrm{H}$ & 4.49932700 & 3.31053400 & -2.66618600 \\
\hline $\mathrm{C}$ & -6.18718900 & -1.28619400 & -0.58223000 & $\mathrm{H}$ & 3.05442000 & 3.82510600 & -1.78206900 \\
\hline $\mathrm{C}$ & -7.49121100 & -0.72607700 & -1.15785500 & $\mathrm{H}$ & 4.42796900 & 4.93170800 & -1.94375400 \\
\hline $\mathrm{H}$ & -7.34038300 & -0.29672100 & -2.15403100 & & & & \\
\hline $\mathrm{H}$ & -8.22585300 & -1.53204100 & -1.25011400 & & & & \\
\hline $\mathrm{H}$ & -7.91985700 & 0.04460000 & -0.50832800 & & & & \\
\hline $\mathrm{C}$ & -5.66094600 & -2.37640700 & -1.53051800 & & & & \\
\hline $\mathrm{H}$ & -4.74673500 & -2.83599500 & -1.14221100 & & & & \\
\hline $\mathrm{H}$ & -6.41052700 & -3.16667200 & -1.64840400 & & & & \\
\hline $\mathrm{H}$ & -5.43931100 & -1.96039000 & -2.51861500 & & & & \\
\hline $\mathrm{C}$ & -6.48638000 & -1.90767500 & 0.79242700 & & & & \\
\hline $\mathrm{H}$ & -5.60009200 & -2.37957200 & 1.22787900 & & & & \\
\hline $\mathrm{H}$ & -6.84560400 & -1.14807100 & 1.49401500 & & & & \\
\hline $\mathrm{H}$ & -7.25885200 & -2.67799100 & 0.69272700 & & & & \\
\hline $\mathrm{C}$ & 6.72469200 & 0.63084300 & -0.64608700 & & & & \\
\hline $\mathrm{C}$ & 6.83339800 & 1.59804300 & 0.54475700 & & & & \\
\hline $\mathrm{H}$ & 7.68924300 & 2.26891500 & 0.40889000 & & & & \\
\hline $\mathrm{H}$ & 5.93484900 & 2.21387300 & 0.64636600 & & & & \\
\hline $\mathrm{H}$ & 6.97242700 & 1.04662400 & 1.48032100 & & & & \\
\hline $\mathrm{C}$ & 6.52722800 & 1.43638500 & -1.94124300 & & & & \\
\hline $\mathrm{H}$ & 5.62046800 & 2.04723300 & -1.90151300 & & & & \\
\hline $\mathrm{H}$ & 7.37934100 & 2.10642700 & -2.10238700 & & & & \\
\hline $\mathrm{H}$ & 6.44628400 & 0.76789300 & -2.80450900 & & & & \\
\hline $\mathrm{C}$ & 8.04038800 & -0.14566700 & -0.75742000 & & & & \\
\hline $\mathrm{H}$ & 8.24727200 & -0.72055800 & 0.15146600 & & & & \\
\hline $\mathrm{H}$ & 8.03091400 & -0.83461400 & -1.60867000 & & & & \\
\hline $\mathrm{H}$ & 8.86767700 & 0.55606400 & -0.90472500 & & & & \\
\hline \multicolumn{4}{|c|}{$\mathbf{2 d}(E=-1727.673628$ Hartree $)$} & \multicolumn{4}{|c|}{$\mathbf{2 e}(E=-1727.671958$ Hartree $)$} \\
\hline $\mathrm{C}$ & 2.46692000 & 2.80975100 & -0.04701500 & $\mathrm{C}$ & 0.49053800 & -4.41355700 & 0.23722100 \\
\hline $\mathrm{C}$ & 1.64802300 & 1.67614000 & -0.12205400 & $\mathrm{C}$ & 0.08370500 & -3.08315900 & 0.31293700 \\
\hline $\mathrm{C}$ & 2.16597700 & 0.38469800 & -0.12454500 & $\mathrm{C}$ & 1.01971700 & -2.04648300 & 0.29128600 \\
\hline $\mathrm{C}$ & 3.57354800 & 0.19427400 & 0.02675500 & $\mathrm{C}$ & 2.40730500 & -2.36751800 & 0.13923900 \\
\hline $\mathrm{C}$ & 4.40703200 & 1.33859400 & 0.08967200 & $\mathrm{C}$ & 2.80361000 & -3.73079200 & 0.08015100 \\
\hline $\mathrm{C}$ & 3.84134200 & 2.62248500 & 0.03773600 & $\mathrm{C}$ & 1.83259900 & -4.73898500 & 0.13846200 \\
\hline $\mathrm{C}$ & 1.39094600 & -0.82630200 & -0.21323700 & $\mathrm{C}$ & 0.72282900 & -0.63442300 & 0.36234200 \\
\hline $\mathrm{C}$ & 4.15364000 & -1.11397800 & 0.15536800 & $\mathrm{C}$ & 3.41309400 & -1.34912300 & 0.01246700 \\
\hline $\mathrm{C}$ & 3.34145100 & -2.28004700 & 0.16212600 & $\mathrm{C}$ & 3.07185200 & 0.02770900 & 0.00670200 \\
\hline $\mathrm{C}$ & 1.92783400 & -2.08003100 & -0.01855300 & $\mathrm{C}$ & 1.67888300 & 0.34251500 & 0.17699400 \\
\hline $\mathrm{C}$ & 3.91332900 & -3.53906000 & 0.32277400 & $\mathrm{C}$ & 4.04994600 & 1.00209500 & -0.14661800 \\
\hline
\end{tabular}




\begin{tabular}{|c|c|c|c|c|c|c|c|}
\hline $\mathrm{H}$ & 3.26601000 & -4.41012500 & 0.32780600 & $\mathrm{H}$ & 3.73063100 & 2.04048700 & -0.14821900 \\
\hline $\mathrm{C}$ & 5.29408400 & -3.66344100 & 0.46773300 & $\mathrm{C}$ & 5.40329900 & 0.66377900 & -0.28937200 \\
\hline $\mathrm{C}$ & 6.10570000 & -2.53762700 & 0.46028500 & $\mathrm{C}$ & 5.74164300 & -0.68649400 & -0.28071400 \\
\hline $\mathrm{C}$ & 5.55763100 & -1.25535300 & 0.31136800 & $\mathrm{C}$ & 4.77783100 & -1.69734400 & -0.13872100 \\
\hline $\mathrm{C}$ & 6.37816500 & -0.07383800 & 0.32648400 & $\mathrm{C}$ & 5.14231500 & -3.08822300 & -0.15523800 \\
\hline $\mathrm{C}$ & 5.82918500 & 1.15968700 & 0.22535900 & $\mathrm{C}$ & 4.19984200 & -4.05432100 & -0.05535300 \\
\hline $\mathrm{H}$ & 6.45492200 & 2.04789000 & 0.25236600 & $\mathrm{H}$ & 4.47769900 & -5.10463700 & -0.08143500 \\
\hline $\mathrm{H}$ & 7.45269600 & -0.19543600 & 0.43457300 & $\mathrm{H}$ & 6.19287500 & -3.34537200 & -0.26156000 \\
\hline $\mathrm{H}$ & 0.57624800 & 1.81405500 & -0.17711200 & $\mathrm{H}$ & -0.25808100 & -5.19913800 & 0.26170100 \\
\hline $\mathrm{H}$ & 4.51369200 & 3.47360700 & 0.08412800 & $\mathrm{H}$ & -0.97189000 & -2.86426400 & 0.39435300 \\
\hline $\mathrm{H}$ & 7.18154700 & -2.63770800 & 0.57727100 & $\mathrm{H}$ & 2.14680700 & -5.77810300 & 0.09164200 \\
\hline $\mathrm{N}$ & 0.95432900 & -3.04466900 & -0.02667100 & $\mathrm{H}$ & 6.77679300 & -0.99410700 & -0.39150200 \\
\hline $\mathrm{C}$ & -0.17926200 & -2.41259700 & -0.21406000 & $\mathrm{~N}$ & 1.12491300 & 1.59418700 & 0.17819500 \\
\hline $\mathrm{N}$ & 0.01931700 & -1.04241200 & -0.33350300 & $\mathrm{C}$ & -0.16299100 & 1.41916900 & 0.34691300 \\
\hline $\mathrm{C}$ & -0.88512100 & -0.15226400 & -0.96760300 & $\mathrm{~N}$ & -0.47888700 & 0.07098900 & 0.45928200 \\
\hline $\mathrm{C}$ & -2.26026300 & -0.06457200 & -0.40445000 & $\mathrm{C}$ & -1.68223200 & -0.42501600 & 1.02115700 \\
\hline $\mathrm{C}$ & -3.08489300 & -1.14080800 & 0.03330300 & $\mathrm{C}$ & -2.96281500 & 0.02033200 & 0.40095700 \\
\hline $\mathrm{C}$ & -1.46560700 & -3.11994600 & -0.30070100 & $\mathrm{C}$ & -3.31806600 & 1.34103800 & 0.00242700 \\
\hline $\mathrm{C}$ & -2.75962900 & -2.56357800 & -0.05563700 & $\mathrm{C}$ & -1.09319000 & 2.55462500 & 0.44870800 \\
\hline $\mathrm{O}$ & -0.48030400 & 0.59899600 & -1.82029800 & $\mathrm{C}$ & -2.49327300 & 2.53533900 & 0.16614800 \\
\hline $\mathrm{C}$ & -3.94525400 & 1.62409600 & 0.17118800 & $\mathrm{O}$ & -1.63468600 & -1.28605900 & 1.86422800 \\
\hline $\mathrm{C}$ & -4.76450200 & 0.59277100 & 0.57653400 & $\mathrm{C}$ & -5.12313200 & -0.90083600 & -0.31192600 \\
\hline $\mathrm{C}$ & -4.38326500 & -0.76197900 & 0.47623000 & $\mathrm{C}$ & -5.49382900 & 0.37729600 & -0.66907800 \\
\hline $\mathrm{C}$ & -2.69915100 & 1.25033800 & -0.35648700 & $\mathrm{C}$ & -4.64706900 & 1.48987900 & -0.48329600 \\
\hline $\mathrm{H}$ & -5.75932600 & 0.79605500 & 0.96107200 & $\mathrm{C}$ & -3.85057900 & -1.03670300 & 0.26650900 \\
\hline $\mathrm{H}$ & -2.03100600 & 2.00935600 & -0.75132500 & $\mathrm{H}$ & -6.47901600 & 0.57203200 & -1.08171200 \\
\hline $\mathrm{C}$ & -5.12451200 & -3.05821900 & 0.54379600 & $\mathrm{H}$ & -3.52691200 & -2.00505300 & 0.63419900 \\
\hline $\mathrm{H}$ & -5.88945200 & -3.81304600 & 0.70176900 & $\mathrm{C}$ & -4.48558800 & 3.89855600 & -0.43662300 \\
\hline $\mathrm{C}$ & -3.82672100 & -3.49560100 & 0.11691900 & $\mathrm{H}$ & -4.91208900 & 4.88929100 & -0.56463400 \\
\hline $\mathrm{C}$ & -5.37736100 & -1.75361100 & 0.76725200 & $\mathrm{C}$ & -3.13127900 & 3.80402700 & 0.02674000 \\
\hline $\mathrm{H}$ & -6.35095400 & -1.41991300 & 1.11410400 & $\mathrm{C}$ & -5.19476000 & 2.79150000 & -0.73303100 \\
\hline $\mathrm{C}$ & -1.32069900 & -4.49033000 & -0.50823100 & $\mathrm{H}$ & -6.21083800 & 2.85795600 & -1.11084900 \\
\hline $\mathrm{C}$ & -3.63512800 & -4.87423200 & -0.08745300 & $\mathrm{C}$ & -0.45172600 & 3.76186400 & 0.71947000 \\
\hline $\mathrm{C}$ & -2.39961600 & -5.36877800 & -0.43250000 & $\mathrm{C}$ & -2.44436500 & 5.00124200 & 0.29775100 \\
\hline $\mathrm{H}$ & -0.32225100 & -4.86570100 & -0.69533700 & $\mathrm{C}$ & -1.12241400 & 4.98259200 & 0.67522300 \\
\hline $\mathrm{H}$ & -4.48626400 & -5.53760400 & 0.03806200 & $\mathrm{H}$ & 0.60922400 & 3.72893700 & 0.93358400 \\
\hline $\mathrm{H}$ & -2.25082400 & -6.42906600 & -0.60734000 & $\mathrm{H}$ & -2.98246900 & 5.93959700 & 0.19650800 \\
\hline $\mathrm{C}$ & -4.33700000 & 3.09958800 & 0.22278800 & $\mathrm{C}$ & 6.44077900 & 1.77806500 & -0.44800100 \\
\hline $\mathrm{C}$ & -5.69440600 & 3.30334900 & 0.90206400 & $\mathrm{C}$ & 6.39624800 & 2.69163300 & 0.78843800 \\
\hline $\mathrm{H}$ & -5.69125700 & 2.92138400 & 1.92864600 & $\mathrm{H}$ & 7.13172000 & 3.49781500 & 0.68803200 \\
\hline $\mathrm{H}$ & -5.92711100 & 4.37198600 & 0.94165600 & $\mathrm{H}$ & 5.41044200 & 3.14849200 & 0.91640000 \\
\hline $\mathrm{H}$ & -6.50048400 & 2.80895700 & 0.34977400 & $\mathrm{H}$ & 6.62683100 & 2.12572600 & 1.69685800 \\
\hline $\mathrm{C}$ & -4.41639800 & 3.64638000 & -1.21290900 & $\mathrm{C}$ & 7.86380800 & 1.22944900 & -0.59084500 \\
\hline $\mathrm{H}$ & -3.45734100 & 3.55456200 & -1.73244500 & $\mathrm{H}$ & 8.16321500 & 0.65176400 & 0.29006500 \\
\hline $\mathrm{H}$ & -5.16957900 & 3.10489800 & -1.79414200 & $\mathrm{H}$ & 7.96249400 & 0.59034100 & -1.47468500 \\
\hline $\mathrm{H}$ & -4.69037200 & 4.70700900 & -1.19561800 & $\mathrm{H}$ & 8.56703100 & 2.06135700 & -0.69987600 \\
\hline $\mathrm{C}$ & -3.27346700 & 3.88645200 & 1.00721700 & $\mathrm{C}$ & 6.11126000 & 2.60244500 & -1.70385300 \\
\hline $\mathrm{H}$ & -3.16758700 & 3.49710300 & 2.02502000 & $\mathrm{H}$ & 6.13651600 & 1.97210200 & -2.59867200 \\
\hline $\mathrm{H}$ & -2.29408200 & 3.83935300 & 0.52055700 & $\mathrm{H}$ & 5.11800500 & 3.05645800 & -1.63899000 \\
\hline $\mathrm{H}$ & -3.56091300 & 4.94160400 & 1.07017100 & $\mathrm{H}$ & 6.84357300 & 3.40811500 & -1.82855400 \\
\hline $\mathrm{H}$ & 5.73657200 & -4.64730200 & 0.58840900 & $\mathrm{H}$ & -0.59276800 & 5.90211000 & 0.90083100 \\
\hline $\mathrm{C}$ & 1.80404600 & 4.18953800 & -0.05623400 & $\mathrm{C}$ & -6.01722500 & -2.12801600 & -0.47842800 \\
\hline $\mathrm{C}$ & 0.90138000 & 4.31936300 & 1.18264000 & $\mathrm{C}$ & -7.35499400 & -1.77333900 & -1.13398200 \\
\hline $\mathrm{H}$ & 0.13224500 & 3.54031600 & 1.20379500 & $\mathrm{H}$ & -7.21255800 & -1.33720900 & -2.12851500 \\
\hline $\mathrm{H}$ & 1.48942700 & 4.23350200 & 2.10220700 & $\mathrm{H}$ & -7.95728000 & -2.67985900 & -1.24913600 \\
\hline $\mathrm{H}$ & 0.39710700 & 5.29268900 & 1.18426500 & $\mathrm{H}$ & -7.92871200 & -1.06778600 & -0.52371600 \\
\hline
\end{tabular}




\begin{tabular}{|c|c|c|c|c|c|c|c|}
\hline $\mathrm{C}$ & 0.95130200 & 4.33942200 & -1.32819600 & $\mathrm{C}$ & -6.29609400 & -2.74396600 & 0.90304500 \\
\hline $\mathrm{H}$ & 0.18147500 & 3.56356700 & -1.39882000 & $\mathrm{H}$ & -5.37364900 & -3.05441000 & 1.40303500 \\
\hline $\mathrm{H}$ & 0.45354700 & 5.31588500 & -1.33423700 & $\mathrm{H}$ & -6.80726200 & -2.02614800 & 1.55243700 \\
\hline $\mathrm{H}$ & 1.57588400 & 4.26490500 & -2.22412100 & $\mathrm{H}$ & -6.93419300 & -3.62803800 & 0.79657900 \\
\hline $\mathrm{C}$ & 2.83202200 & 5.32441100 & -0.02740900 & $\mathrm{C}$ & -5.29730300 & -3.15942800 & -1.36368800 \\
\hline $\mathrm{H}$ & 3.44342700 & 5.29104200 & 0.88050300 & $\mathrm{H}$ & -5.08722800 & -2.74364400 & -2.35437500 \\
\hline $\mathrm{H}$ & 3.49830800 & 5.28376500 & -0.89557200 & $\mathrm{H}$ & -4.34810700 & -3.47842800 & -0.92211800 \\
\hline $\mathrm{H}$ & 2.31479400 & 6.28913400 & -0.04639200 & $\mathrm{H}$ & -5.92458100 & -4.04867900 & -1.48896000 \\
\hline \multicolumn{4}{|c|}{$\mathbf{2 f}(E=-1727.673076$ Hartree $)$} & \multicolumn{4}{|c|}{$\mathbf{5}(E=-1651.463680$ Hartree $)$} \\
\hline $\mathrm{C}$ & 4.43180300 & 1.20421100 & -0.16608900 & $\mathrm{C}$ & -2.05071700 & -4.82832300 & -0.20530000 \\
\hline $\mathrm{C}$ & 3.04385200 & 1.01370000 & -0.16843200 & $\mathrm{C}$ & -1.51995100 & -3.55670300 & -0.24045200 \\
\hline $\mathrm{C}$ & 2.46492200 & -0.24975000 & -0.08890100 & $\mathrm{C}$ & -2.35056000 & -2.42050200 & -0.15946200 \\
\hline $\mathrm{C}$ & 3.30830000 & -1.39455800 & 0.06519800 & $\mathrm{C}$ & -3.75591300 & -2.58968600 & 0.00830600 \\
\hline $\mathrm{C}$ & 4.71291100 & -1.20561900 & 0.06372400 & $\mathrm{C}$ & -4.25986100 & -3.90565100 & 0.02695900 \\
\hline $\mathrm{C}$ & 5.24831400 & 0.08541600 & -0.06326700 & $\mathrm{C}$ & -3.43446600 & -5.00507100 & -0.08477000 \\
\hline $\mathrm{C}$ & 1.04928700 & -0.53025200 & -0.10588600 & $\mathrm{C}$ & -1.87325800 & -1.06163600 & -0.17019200 \\
\hline $\mathrm{C}$ & 2.77533900 & -2.71560900 & 0.25290800 & $\mathrm{C}$ & -4.64446500 & -1.43641000 & 0.20505900 \\
\hline $\mathrm{C}$ & 1.37420800 & -2.93805900 & 0.31309300 & $\mathrm{C}$ & -4.10364600 & -0.13329100 & 0.29545100 \\
\hline $\mathrm{C}$ & 0.53190800 & -1.78574200 & 0.13405300 & $\mathrm{C}$ & -2.68841500 & 0.01275300 & 0.10793200 \\
\hline $\mathrm{C}$ & 0.86835000 & -4.21776500 & 0.52498800 & $\mathrm{C}$ & -4.92583100 & 0.98084700 & 0.53524400 \\
\hline $\mathrm{H}$ & -0.20744000 & -4.35322700 & 0.57038300 & $\mathrm{H}$ & -4.45885300 & 1.95831100 & 0.60134300 \\
\hline $\mathrm{C}$ & 1.73997500 & -5.29553600 & 0.67019600 & $\mathrm{C}$ & -6.28776300 & 0.81921300 & 0.67243900 \\
\hline $\mathrm{C}$ & 3.11285000 & -5.10106100 & 0.61118100 & $\mathrm{C}$ & -6.84620200 & -0.46408100 & 0.56832200 \\
\hline $\mathrm{C}$ & 3.65145300 & -3.82215000 & 0.40874900 & $\mathrm{C}$ & -6.04277100 & -1.56396400 & 0.34159700 \\
\hline $\mathrm{C}$ & 5.07115000 & -3.59678800 & 0.36609700 & $\mathrm{H}$ & -0.44854100 & -3.43530600 & -0.32448800 \\
\hline $\mathrm{C}$ & 5.57438800 & -2.35042900 & 0.20556300 & $\mathrm{H}$ & -3.85855000 & -6.00387000 & -0.06229100 \\
\hline $\mathrm{H}$ & 6.64839800 & -2.18527800 & 0.18594800 & $\mathrm{H}$ & -7.91910500 & -0.59708200 & 0.66669100 \\
\hline $\mathrm{H}$ & 5.73190200 & -4.45243400 & 0.47696300 & $\mathrm{~N}$ & -1.97591900 & 1.18145800 & 0.16693800 \\
\hline $\mathrm{H}$ & 2.40549100 & 1.88289700 & -0.24393100 & $\mathrm{C}$ & -0.72747300 & 0.85462900 & -0.06045200 \\
\hline $\mathrm{H}$ & 6.32917100 & 0.18643500 & -0.06653800 & $\mathrm{~N}$ & -0.59197700 & -0.51329800 & -0.26940500 \\
\hline $\mathrm{H}$ & 3.79052900 & -5.94264400 & 0.72780300 & $\mathrm{C}$ & 0.50371000 & -1.11509100 & -0.94049700 \\
\hline $\mathrm{N}$ & -0.83692500 & -1.76359300 & 0.17521200 & $\mathrm{C}$ & 1.86313200 & -0.89085700 & -0.37226500 \\
\hline $\mathrm{C}$ & -1.18390800 & -0.51483800 & -0.02089500 & $\mathrm{C}$ & 2.40469200 & 0.33280100 & 0.11730200 \\
\hline $\mathrm{N}$ & -0.07076800 & 0.29778000 & -0.19390600 & $\mathrm{C}$ & 0.34403300 & 1.86236700 & -0.11177400 \\
\hline $\mathrm{C}$ & -0.10086100 & 1.58568700 & -0.78590600 & $\mathrm{C}$ & 1.73760300 & 1.63005800 & 0.10272700 \\
\hline $\mathrm{C}$ & -0.98188100 & 2.60864000 & -0.15540000 & $\mathrm{O}$ & 0.29423200 & -1.89390000 & -1.83734800 \\
\hline $\mathrm{C}$ & -2.32482200 & 2.43740600 & 0.28727500 & $\mathrm{C}$ & 3.91977300 & -2.13481400 & 0.12492200 \\
\hline $\mathrm{C}$ & -2.59338200 & -0.09786500 & -0.09279000 & $\mathrm{C}$ & 4.46743700 & -0.95280100 & 0.57317800 \\
\hline $\mathrm{C}$ & -3.10642300 & 1.20871100 & 0.17460400 & $\mathrm{C}$ & 3.76260500 & 0.26883700 & 0.53775500 \\
\hline $\mathrm{O}$ & 0.67873800 & 1.85695000 & -1.66567100 & $\mathrm{C}$ & 2.61325000 & -2.05703900 & -0.38545500 \\
\hline $\mathrm{C}$ & -0.96684200 & 4.94742000 & 0.50975100 & $\mathrm{H}$ & 5.48735700 & -0.92174300 & 0.94423200 \\
\hline $\mathrm{C}$ & -2.27556900 & 4.82659800 & 0.91158400 & $\mathrm{H}$ & 2.14968900 & -2.93858100 & -0.81645600 \\
\hline $\mathrm{C}$ & -2.97434800 & 3.61190200 & 0.77064800 & $\mathrm{C}$ & 2.53226100 & 2.79032300 & 0.32240400 \\
\hline $\mathrm{C}$ & -0.34142300 & 3.83916300 & -0.06284800 & $\mathrm{C}$ & -0.13403000 & 3.16032500 & -0.24570100 \\
\hline $\mathrm{H}$ & -2.81005200 & 5.68076800 & 1.31824900 & $\mathrm{C}$ & 1.99750100 & 4.08742600 & 0.19261400 \\
\hline $\mathrm{H}$ & 0.66741000 & 3.92255500 & -0.45271200 & $\mathrm{C}$ & 0.67383000 & 4.30117300 & -0.12685700 \\
\hline $\mathrm{C}$ & -5.12411100 & 2.51371700 & 0.80545500 & $\mathrm{H}$ & -1.19933700 & 3.26962500 & -0.41284600 \\
\hline $\mathrm{H}$ & -6.19873600 & 2.51858500 & 0.96363000 & $\mathrm{H}$ & 2.67334200 & 4.92107200 & 0.35615900 \\
\hline $\mathrm{C}$ & -4.51581400 & 1.29596200 & 0.35195200 & $\mathrm{H}$ & -5.32326900 & -4.07133700 & 0.15091500 \\
\hline $\mathrm{C}$ & -4.37888800 & 3.60788800 & 1.05850300 & $\mathrm{H}$ & -6.51380800 & -2.53675200 & 0.26401600 \\
\hline $\mathrm{H}$ & -4.82974700 & 4.52333700 & 1.43024500 & $\mathrm{H}$ & -6.92617100 & 1.67796200 & 0.85468400 \\
\hline $\mathrm{C}$ & -3.46464500 & -1.15860200 & -0.31045800 & $\mathrm{H}$ & -1.39253700 & -5.68906100 & -0.26801400 \\
\hline $\mathrm{C}$ & -5.35807000 & 0.18835500 & 0.13164700 & $\mathrm{C}$ & 4.48059200 & 1.46285500 & 0.87447200 \\
\hline $\mathrm{C}$ & -4.86066000 & -1.04390400 & -0.23510600 & $\mathrm{C}$ & 3.90466000 & 2.67244500 & 0.72135700 \\
\hline $\mathrm{H}$ & -3.01311300 & -2.12315300 & -0.51184500 & $\mathrm{H}$ & 5.51237900 & 1.36806300 & 1.20026800 \\
\hline $\mathrm{H}$ & -6.42433500 & 0.34148600 & 0.26592600 & $\mathrm{H}$ & 4.45908200 & 3.58579600 & 0.91726400 \\
\hline
\end{tabular}




\begin{tabular}{|c|c|c|c|c|c|c|c|}
\hline $\mathrm{H}$ & 1.34211900 & -6.29257100 & 0.83152500 & $\mathrm{C}$ & 4.65548200 & -3.47343500 & 0.12788100 \\
\hline $\mathrm{C}$ & 4.97380500 & 2.63007900 & -0.29299800 & $\mathrm{C}$ & 6.05814400 & -3.35120000 & 0.73024000 \\
\hline $\mathrm{C}$ & 4.46236900 & 3.47775600 & 0.88417600 & $\mathrm{H}$ & 6.02165100 & -2.99821000 & 1.76641600 \\
\hline $\mathrm{H}$ & 3.36903600 & 3.53028300 & 0.90090300 & $\mathrm{H}$ & 6.54403800 & -4.33188000 & 0.72748600 \\
\hline $\mathrm{H}$ & 4.79385800 & 3.05727100 & 1.83912900 & $\mathrm{H}$ & 6.68701400 & -2.66629000 & 0.15158700 \\
\hline $\mathrm{H}$ & 4.84631300 & 4.50110300 & 0.80577000 & $\mathrm{C}$ & 3.85285300 & -4.48776800 & 0.96014300 \\
\hline $\mathrm{C}$ & 4.47879700 & 3.24011800 & -1.61609200 & $\mathrm{H}$ & 2.85023900 & -4.64358400 & 0.54986800 \\
\hline $\mathrm{H}$ & 3.38551100 & 3.25228200 & -1.67452100 & $\mathrm{H}$ & 4.36588700 & -5.45562800 & 0.96861200 \\
\hline $\mathrm{H}$ & 4.83887400 & 4.27059300 & -1.71428500 & $\mathrm{H}$ & 3.74607100 & -4.14530100 & 1.99445800 \\
\hline $\mathrm{H}$ & 4.84941800 & 2.66276700 & -2.46920000 & $\mathrm{C}$ & 4.78530500 & -3.98464300 & -1.31694500 \\
\hline $\mathrm{C}$ & 6.50489800 & 2.66822100 & -0.28417100 & $\mathrm{H}$ & 3.80740100 & -4.12851300 & -1.78628700 \\
\hline $\mathrm{H}$ & 6.91250500 & 2.26705100 & 0.64989000 & $\mathrm{H}$ & 5.35337000 & -3.27725100 & -1.92946800 \\
\hline $\mathrm{H}$ & 6.92549600 & 2.09799900 & -1.11907900 & $\mathrm{H}$ & 5.30788800 & -4.94741300 & -1.32778300 \\
\hline $\mathrm{H}$ & 6.84649000 & 3.70377900 & -0.38127900 & $\mathrm{C}$ & 0.05550300 & 5.68629700 & -0.30864900 \\
\hline $\mathrm{H}$ & -0.43637000 & 5.88887500 & 0.60266200 & $\mathrm{C}$ & 1.09313600 & 6.80071100 & -0.14512800 \\
\hline $\mathrm{C}$ & -5.73471100 & -2.26580100 & -0.51187900 & $\mathrm{H}$ & 0.61396900 & 7.77258500 & -0.29965600 \\
\hline $\mathrm{C}$ & -7.22615800 & -1.94079700 & -0.38810900 & $\mathrm{H}$ & 1.52984500 & 6.80077400 & 0.85930100 \\
\hline $\mathrm{H}$ & -7.81542800 & -2.83568100 & -0.61146000 & $\mathrm{H}$ & 1.90326700 & 6.70639100 & -0.87621600 \\
\hline $\mathrm{H}$ & -7.48561400 & -1.61605500 & 0.62519900 & $\mathrm{C}$ & -0.55008900 & 5.78471100 & -1.71885700 \\
\hline $\mathrm{H}$ & -7.52678900 & -1.15720200 & -1.09188100 & $\mathrm{H}$ & 0.22060100 & 5.64431000 & -2.48370900 \\
\hline $\mathrm{C}$ & -5.45997400 & -2.76691900 & -1.93964000 & $\mathrm{H}$ & -1.32631900 & 5.03015000 & -1.87750800 \\
\hline $\mathrm{H}$ & -5.70013900 & -1.99353600 & -2.67642100 & $\mathrm{H}$ & -1.00413500 & 6.77112200 & -1.86415400 \\
\hline $\mathrm{H}$ & -4.41119200 & -3.04833500 & -2.07327200 & $\mathrm{C}$ & -1.05153400 & 5.89050000 & 0.73955800 \\
\hline $\mathrm{H}$ & -6.07505500 & -3.64820100 & -2.15228700 & $\mathrm{H}$ & -1.84236200 & 5.14044600 & 0.64462500 \\
\hline $\mathrm{C}$ & -5.38979800 & -3.37548300 & 0.49592800 & $\mathrm{H}$ & -0.64307600 & 5.82421900 & 1.75312700 \\
\hline $\mathrm{H}$ & -4.33988700 & -3.67488000 & 0.42506100 & $\mathrm{H}$ & -1.50695200 & 6.87922400 & 0.61524200 \\
\hline $\mathrm{H}$ & -5.57712400 & -3.04026400 & 1.52113100 & & & & \\
\hline $\mathrm{H}$ & -6.00710100 & -4.26007400 & 0.30410400 & & & & \\
\hline \multicolumn{4}{|c|}{$4(E=-1337.315930$ Hartree $)$} & \multicolumn{4}{|c|}{ IM-5 ( $E=-1489.713333$ Hartree $)$} \\
\hline $\mathrm{H}$ & -1.90574600 & 4.52250400 & -1.40978200 & $\mathrm{H}$ & 2.51499200 & 4.19908700 & -2.23841200 \\
\hline $\mathrm{C}$ & -2.30935700 & 3.56282500 & -1.10350400 & $\mathrm{C}$ & 2.74672200 & 3.24209800 & -1.78121400 \\
\hline $\mathrm{C}$ & -3.38555200 & 1.06904000 & -0.31157000 & $\mathrm{C}$ & 3.35232900 & 0.76390600 & -0.60018100 \\
\hline $\mathrm{C}$ & -1.47576000 & 2.47429200 & -0.95867200 & $\mathrm{C}$ & 1.72924200 & 2.31439600 & -1.56735300 \\
\hline $\mathrm{C}$ & -3.68321200 & 3.42266900 & -0.85526800 & $\mathrm{C}$ & 4.05408900 & 2.94993500 & -1.41677900 \\
\hline $\mathrm{C}$ & -4.20658300 & 2.20432600 & -0.46940200 & $\mathrm{C}$ & 4.37767100 & 1.72003700 & -0.82685300 \\
\hline $\mathrm{C}$ & -2.00070500 & 1.23192900 & -0.56503500 & $\mathrm{C}$ & 2.01857000 & 1.08249400 & -0.98339200 \\
\hline $\mathrm{H}$ & -0.40892400 & 2.55104800 & -1.14865800 & $\mathrm{H}$ & 0.70347300 & 2.52738800 & -1.85199500 \\
\hline $\mathrm{H}$ & -4.34328500 & 4.27680000 & -0.96863200 & $\mathrm{H}$ & 4.84539300 & 3.67517200 & -1.58641900 \\
\hline $\mathrm{H}$ & -5.27319400 & 2.13542700 & -0.28928300 & $\mathrm{C}$ & 3.67527400 & -0.49869800 & 0.00946500 \\
\hline $\mathrm{C}$ & -3.92066600 & -0.23914800 & 0.09505100 & $\mathrm{C}$ & 4.34141100 & -2.95715000 & 1.20518800 \\
\hline $\mathrm{C}$ & -4.92458500 & -2.77387600 & 0.86889000 & $\mathrm{C}$ & 2.67439600 & -1.48442600 & 0.25739300 \\
\hline $\mathrm{C}$ & -3.06324600 & -1.36398300 & 0.23549800 & $\mathrm{C}$ & 5.01756300 & -0.77955200 & 0.37755700 \\
\hline $\mathrm{C}$ & -5.28976200 & -0.44128400 & 0.36000200 & $\mathrm{C}$ & 5.32849200 & -2.01054200 & 0.97216500 \\
\hline $\mathrm{C}$ & -5.78433500 & -1.67444800 & 0.73777500 & $\mathrm{C}$ & 3.01976100 & -2.69857100 & 0.85026300 \\
\hline $\mathrm{C}$ & -3.57805800 & -2.61515400 & 0.61914900 & $\mathrm{H}$ & 6.35882600 & -2.21480800 & 1.25007300 \\
\hline $\mathrm{H}$ & -5.98431700 & 0.38546800 & 0.26851200 & $\mathrm{H}$ & 2.25497400 & -3.44753100 & 1.03631100 \\
\hline $\mathrm{H}$ & -6.84546900 & -1.79031300 & 0.93319000 & $\mathrm{H}$ & 4.59785100 & -3.90523600 & 1.66701700 \\
\hline $\mathrm{H}$ & -2.90593100 & -3.46397000 & 0.71784200 & $\mathrm{C}$ & 1.01479700 & 0.08357700 & -0.72505700 \\
\hline $\mathrm{H}$ & -5.31502300 & -3.74223900 & 1.16448200 & $\mathrm{C}$ & 1.34482600 & -1.12558600 & -0.13933900 \\
\hline $\mathrm{C}$ & -1.15805000 & 0.08091300 & -0.40500300 & $\mathrm{~N}$ & -0.33640500 & 0.16376800 & -0.96101700 \\
\hline $\mathrm{C}$ & -1.67510200 & -1.14224800 & -0.03285800 & $\mathrm{~N}$ & 0.15768100 & -1.80798900 & -0.04594200 \\
\hline $\mathrm{N}$ & 0.19971700 & -0.01020000 & -0.58232800 & $\mathrm{H}$ & -0.02963600 & -2.62477000 & 0.52039200 \\
\hline $\mathrm{N}$ & -0.59186500 & -1.99379400 & 0.00520100 & $\mathrm{C}$ & -0.82312300 & -0.98206100 & -0.53425900 \\
\hline $\mathrm{H}$ & -0.58212000 & -2.95928800 & 0.30029200 & $\mathrm{C}$ & -2.22185300 & -1.43395300 & -0.66724800 \\
\hline $\mathrm{C}$ & 0.51371600 & -1.26215500 & -0.32385500 & $\mathrm{C}$ & -4.75329600 & -2.25803500 & -1.51272800 \\
\hline $\mathrm{C}$ & 1.87047600 & -1.81206200 & -0.44019500 & $\mathrm{C}$ & -3.34118800 & -0.60273600 & -0.37135700 \\
\hline
\end{tabular}




\begin{tabular}{|c|c|c|c|c|c|c|c|}
\hline $\mathrm{C}$ & 4.45474900 & -2.75580900 & -0.87958200 & $\mathrm{C}$ & -2.41096300 & -2.67121000 & -1.26184600 \\
\hline $\mathrm{C}$ & 2.96542800 & -1.07883600 & 0.04916800 & $\mathrm{C}$ & -3.68591800 & -3.11293100 & -1.64344900 \\
\hline $\mathrm{C}$ & 2.07668300 & -3.01726800 & -1.11696400 & $\mathrm{C}$ & -4.59396200 & -0.99049400 & -0.91239800 \\
\hline $\mathrm{C}$ & 3.36453500 & -3.49488500 & -1.32920300 & $\mathrm{H}$ & -1.54110200 & -3.27735900 & -1.49777500 \\
\hline $\mathrm{C}$ & 4.25248000 & -1.56123100 & -0.19369500 & $\mathrm{H}$ & -3.80835900 & -4.08849500 & -2.10210500 \\
\hline $\mathrm{H}$ & 1.22216700 & -3.55790200 & -1.51568200 & $\mathrm{H}$ & -5.73541600 & -2.53544700 & -1.88556800 \\
\hline $\mathrm{H}$ & 3.51573800 & -4.42703800 & -1.86367200 & $\mathrm{C}$ & -3.28638200 & 0.63228400 & 0.40484500 \\
\hline $\mathrm{H}$ & 5.10007400 & -0.99362200 & 0.17874700 & $\mathrm{C}$ & -3.20987600 & 3.16695700 & 1.68358100 \\
\hline $\mathrm{H}$ & 5.46483600 & -3.11098600 & -1.05677100 & $\mathrm{C}$ & -2.26815600 & 0.95461700 & 1.34547000 \\
\hline $\mathrm{C}$ & 2.80634700 & 0.12296600 & 0.92952700 & $\mathrm{C}$ & -4.34778300 & 1.56112300 & 0.26629500 \\
\hline $\mathrm{C}$ & 2.74197300 & 2.26911300 & 2.73453300 & $\mathrm{C}$ & -4.27419300 & 2.82968700 & 0.88461300 \\
\hline $\mathrm{C}$ & 2.99785700 & 1.43540400 & 0.47779000 & $\mathrm{C}$ & -2.22504300 & 2.20100600 & 1.94466400 \\
\hline $\mathrm{C}$ & 2.56761700 & -0.09253300 & 2.28940900 & $\mathrm{H}$ & -5.09308600 & 3.52723200 & 0.73075000 \\
\hline $\mathrm{C}$ & 2.51957100 & 0.97092600 & 3.18518300 & $\mathrm{H}$ & -1.45229300 & 2.41005100 & 2.67924900 \\
\hline $\mathrm{C}$ & 2.99188100 & 2.49417200 & 1.38600800 & $\mathrm{H}$ & -3.15728900 & 4.14062000 & 2.15899800 \\
\hline $\mathrm{H}$ & 2.43476600 & -1.11110000 & 2.64384800 & $\mathrm{C}$ & -1.35491700 & -0.08304900 & 1.93532500 \\
\hline $\mathrm{H}$ & 2.32531900 & 0.78215800 & 4.23644500 & $\mathrm{O}$ & -1.72351200 & -1.16918300 & 2.29964400 \\
\hline $\mathrm{H}$ & 3.17989100 & 3.49575000 & 1.01188000 & $\mathrm{O}$ & -0.07913100 & 0.30683800 & 2.13621000 \\
\hline $\mathrm{H}$ & 2.72369300 & 3.10154200 & 3.43065500 & $\mathrm{H}$ & 0.11487500 & 1.08254500 & 1.58345300 \\
\hline $\mathrm{C}$ & 3.21306300 & 1.78323500 & -0.97394700 & $\mathrm{C}$ & 5.72745000 & 1.40048600 & -0.44158000 \\
\hline $\mathrm{O}$ & 4.11044600 & 2.50625700 & -1.32815600 & $\mathrm{H}$ & 6.50110400 & 2.14198500 & -0.62256100 \\
\hline $\mathrm{O}$ & 2.32657800 & 1.28039200 & -1.83242200 & $\mathrm{C}$ & 6.03183200 & 0.21228600 & 0.13056000 \\
\hline \multirow[t]{5}{*}{$\mathrm{H}$} & 1.55796200 & 0.85917300 & -1.36427800 & $\mathrm{H}$ & 7.05401900 & -0.01903700 & 0.41782500 \\
\hline & & & & $\mathrm{C}$ & -5.54507400 & 1.18684300 & -0.43302800 \\
\hline & & & & $\mathrm{H}$ & -6.34974600 & 1.91349700 & -0.50052700 \\
\hline & & & & $\mathrm{C}$ & -5.69288900 & -0.06533200 & -0.91901400 \\
\hline & & & & $\mathrm{H}$ & -6.62521800 & -0.38102700 & -1.37869500 \\
\hline
\end{tabular}




\section{X-ray Structure of $\left[2 b \cdot(T F A)_{2}\right]$}
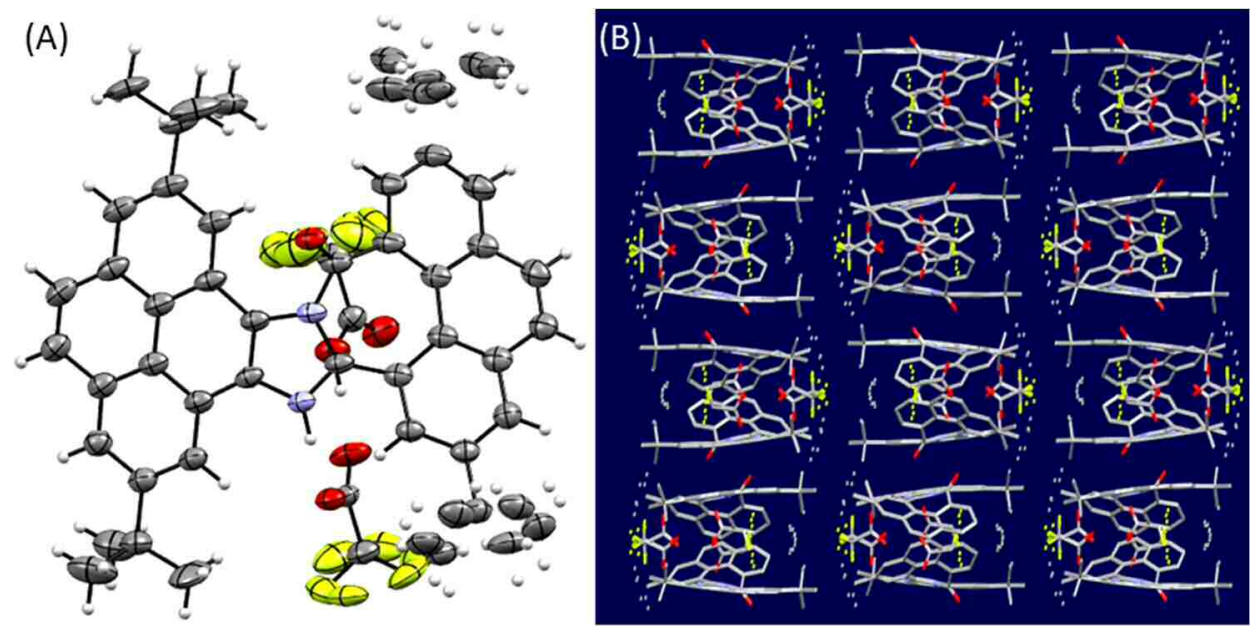

Fig. S-23. (A) ORTEP drawing (50\% probability), and (B) crystal packing diagrams of the complex of $\left[\mathbf{2 b} \cdot(\mathrm{TFA})_{2}\right]$. Hydrogen atoms are not shown for clarity. 